HU-EP-03/36

\title{
General chiral gauge theories on the lattice
}

\author{
Werner Kerler \\ Institut für Physik, Humboldt-Universität, D-12489 Berlin, Germany
}

\begin{abstract}
We still extend the large class of Dirac operators decribing massless fermions on the lattice found recently, only requiring that such operators decompose into Weyl operators. After deriving general relations and constructions of operators, we study the basis representations of the chiral projections. We then investigate correlation functions of Weyl fermions for any value of the index, stressing the related conditions for basis transformations and their consequences, and getting the precise behaviors under gauge transformations and $\mathrm{CP}$ transformations. Various further developments include considerations of the explicit form of the effective action and of a representation of the general correlation functions in terms of alternating multilinear forms. For comparison we also consider gauge-field variations and their respective applications. Finally we compare with continuum perturbation theory.
\end{abstract}

\section{Introduction}

We reconsider chiral gauge theories on the lattice generalizing the basic structure which has been introduced in the overlap formalism of Narayanan and Neuberger [1] and in the formulation of Lüscher 2]. The main aim of the generalization is to reveal the really relevant features and thereby to allow further developments of the subject.

Massless Dirac operators $D$ on the lattice are functions of a basic unitary operator $V$. The simplest case of this are Ginsparg-Wilson (GW) fermions [3] for which $D$ is $\mathbb{1}-V$ times a constant. More generally this holds for the large class of operators [4] where $D=F(V)$ satisfies $D+D^{\dagger} V=0$. In addition to GW fermions this class includes the ones proposed by Fujikawa [5] and the extension of the latter [6] as special cases. Here we go still further, only requiring that $D$ allows a decomposition into Weyl operators.

The chiral projections for this decomposition, which are implicit in Ref. [1] and formulated in Ref. [2] in the GW case, are $P_{-}=\frac{1}{2}\left(1-\gamma_{5} V\right)$ and $\bar{P}_{+}=\frac{1}{2}\left(1+\gamma_{5}\right)$. These 
forms have turned out to be suitable for the general class of operators in Ref. [4, too. In the GW case Hasenfratz [7] has pointed that, instead of $\gamma_{5} V$ and $\gamma_{5}$, one could use $\gamma_{5}((1-s) \mathbb{1}+s V) / \mathcal{N}$ and $(s \mathbb{1}+(1-s) V) \gamma_{5} / \mathcal{N}$, respectively, with a parameter $s$. We here more generally introduce $\gamma_{5} G(V)$ and $\bar{G}(V) \gamma_{5}$, respectively, with appropriate functions $G(V)$ and $\bar{G}(V)$, which leads to the more general requirement $D+D^{\dagger} \bar{G} G=0$ for $D$.

Starting from the spectral representation of $V$ we first determine basic conditions on $D$ and relations for its index. We then get the details of the Weyl-operator decomposition and find that $G$ and $\bar{G}$ must be generally different. Next from the spectral representations of the chiral projections we obtain detailed information about their structure. Our general construction of Dirac operators [4] as well as the related realizations of $V$ are seen to extend to the larger class of operators here.

After making sure about the transformation properties of our general operators and a study of basis representations of chiral projections, which includes basis transformations, (finite) gauge transformations and CP transformtions, we give a formulation of the correlation functions of Weyl fermions for any value of the index. The additional conditions, which follow from the requirement that these functions must remain invariant under basis transformations, are carefully discussed. The crucial meaning of the emerging decomposition of the total set of bases into subsets is stressed.

Considering gauge transformations the importance of finite transformations in the analysis becomes apparent. The general fermionic correlation functions exhibit gauge-covariant behavior of the fermion fields. In the exceptional cases, where either $G$ or $\bar{G}$ equals the identity, in addition constant phase factors occur. The behavior under CP transformations turns out to differ from that of continuum theory by an interchange of $G$ and $\bar{G}$, where the interchanged choice is a legitimate one, too. The effects of this interchange are also discussed.

Turning to further aspects we derive the explicit form of the effective action, give a formulation not referring to bases and address locality properties. Using the spectral representations we get a form of the correlation functions for general index and zero modes with a reduced chiral determinant. We next observe that the general correlation functions can be reformulated so that they are completely determined by alternating multilinear forms and $D$. Particular features of this representation are pointed out.

Also considering variations of the gauge field we discuss their application to specify basisindependent quantities. The properties of the variations related to gauge transformations are obtained from those of the finite transformations and are seen to rely entirely on the latter. Considerations of variations of the effective action allow various comparisons.

The developments in Ref. [2] and the investigations of CP properties in Ref. 6] are discussed in the light of our results. A comparison with continuum perturbation theory includes the discussion of related Ward-Takahashi identities and the derivation of perturbative results on the basis of the present nonperturbative definitions.

In Section 2 we introduce basic conditions and general relations. In Section 3 we describe the realizations of the operators. Transformation properties of operators are considered in Section 4 and basis representations of the chiral projections are studied in Section 5. 
In Section 6 we investigate the properties of correlation functions. Section 7 describes the alternating-form representation. Gauge-field variations are considered in Section 8. Section 9 is devoted to discussions of literature. The comparison with continuum perturbation theory is performed in Section 10. Section 11 contains our conclusions.

\section{General conditions and relations}

\subsection{Basic unitary operator}

We consider a finite lattice with periodic boundary conditions and dimensionless quantities. Throughout we make consequent use of the fact that the operators describing fermions can be considered as acting in a unitary space of finite dimension.

Imposing the conditions

$$
V^{\dagger}=V^{-1}=\gamma_{5} V \gamma_{5}
$$

on $V$ we obtain the spectral representation

$$
V=P_{1}^{(+)}+P_{1}^{(-)}-P_{2}^{(+)}-P_{2}^{(-)}+\sum_{k\left(0<\varphi_{k}<\pi\right)}\left(\mathrm{e}^{i \varphi_{k}} P_{k}^{(\mathrm{I})}+\mathrm{e}^{-i \varphi_{k}} P_{k}^{(\mathrm{II})}\right)
$$

in which the orthogonal projections satisfy

$$
\gamma_{5} P_{j}^{( \pm)}=P_{j}^{( \pm)} \gamma_{5}= \pm P_{j}^{( \pm)}, \quad \gamma_{5} P_{k}^{(\mathrm{I})}=P_{k}^{(\mathrm{II})} \gamma_{5}
$$

The dimensions of the right-handed and left-handed eigenspaces are $N_{ \pm}(1)=\operatorname{Tr} P_{1}^{( \pm)}$for eigenvalue 1 and $N_{ \pm}(-1)=\operatorname{Tr} P_{2}^{( \pm)}$for eigenvalue -1 . From (2.3) one gets $N_{k}=\operatorname{Tr} P_{k}^{(\mathrm{I})}=$ $\operatorname{Tr} P_{k}^{(\mathrm{II})}$ for the dimensions of the other eigenspaces and the relations

$$
\begin{aligned}
& \operatorname{Tr}\left(\gamma_{5} P_{1}^{( \pm)}\right)= \pm N_{ \pm}(1), \quad \operatorname{Tr}\left(\gamma_{5} P_{2}^{( \pm)}\right)= \pm N_{ \pm}(-1), \\
& \operatorname{Tr}\left(\gamma_{5} P_{k}^{(\mathrm{I})}\right)=\operatorname{Tr}\left(\gamma_{5} P_{k}^{(\mathrm{II})}\right)=0 .
\end{aligned}
$$

With this we obtain

$$
\lim _{\zeta \rightarrow 0} \operatorname{Tr}\left(\gamma_{5} \frac{-\zeta}{V-\mathbb{1}-\zeta \mathbb{1}}\right)=N_{+}(1)-N_{-}(1)
$$

and also find

$$
\lim _{\zeta \rightarrow 0} \operatorname{Tr}\left(\gamma_{5} \frac{V-\mathbb{1}}{V-\mathbb{1}-\zeta \mathbb{1}}\right)=N_{+}(-1)-N_{-}(-1) .
$$

Addition up these relations gives the sum rule

$$
N_{+}(1)-N_{-}(1)+N_{+}(-1)-N_{-}(-1)=0 .
$$

The spectral representation (2.2) with (2.3) also gives

$$
\operatorname{Tr}\left(\gamma_{5} V\right)=N_{+}(1)-N_{-}(1)-N_{+}(-1)+N_{-}(-1) .
$$

Combining (2.8) and (2.7) we have

$$
N_{+}(1)-N_{-}(1)=\frac{1}{2} \operatorname{Tr}\left(\gamma_{5} V\right) .
$$




\subsection{Dirac operator}

With (2.2) the spectral representation of $D=F(V)$ becomes

$$
\begin{aligned}
D= & f(1)\left(P_{1}^{(+)}+P_{1}^{(-)}\right)+f(-1)\left(P_{2}^{(+)}+P_{2}^{(-)}\right) \\
& +\sum_{k\left(0<\varphi_{k}<\pi\right)}\left(f\left(\mathrm{e}^{i \varphi_{k}}\right) P_{k}^{(\mathrm{I})}+f\left(\mathrm{e}^{-i \varphi_{k}}\right) P_{k}^{(\mathrm{II})}\right),
\end{aligned}
$$

in which $D$ is characterized by the spectral function $f\left(\mathrm{e}^{i \varphi}\right)$. On the latter we impose three conditions. Firstly we require

$$
f^{*}(v)=f\left(v^{*}\right),
$$

by which $D$ gets $\gamma_{5}$-Hermitian, $\gamma_{5} D \gamma_{5}=D^{\dagger}$. Conversely $\gamma_{5}$-Hermiticity of $D$ implies (2.11). According to (2.11) obviously $f(1)$ and $f(-1)$ are real. Secondly imposing

$$
f(1)=0,
$$

since the eigenvalue 1 of $V$ corresponds to the eigenvalue 0 of $D$, one describes massless fermions. Thirdly we then must have

$$
f(-1) \neq 0
$$

to allow for a nonvanishing index of $D$. This is seen noting that the index is given by

$$
I=\lim _{\zeta \rightarrow 0} \operatorname{Tr}\left(\gamma_{5} \frac{-\zeta}{D-\zeta \mathbb{1}}\right),
$$

which for $f(-1)=0$ would give $N_{+}(1)-N_{-}(1)+N_{+}(-1)-N_{-}(-1)$ and thus according to (2.7) would be always vanishing.

With the conditions on $f$ in place we obtain for the index of $D$

$$
I=N_{+}(1)-N_{-}(1) .
$$

With this (2.7) tells that a nonvanishing value of $I$ requires the the occurrence of the eigenvalue -1 of $V$ in addition to the eigenvalue 1 of $V$. Thus (2.7) is seen to corresponds to the sum rule found by Chiu $[8]$ in the GW case. Further with (2.9) and (2.15) we have

$$
I=\frac{1}{2} \operatorname{Tr}\left(\gamma_{5} V\right),
$$

which generalizes results of the overlap formalism [1] and of the GW case [9, 10].

\subsection{Weyl operator decomposition}

We define chiral projections by

$$
P_{ \pm}=P_{ \pm}^{\dagger}=\frac{1}{2}\left(\mathbb{1} \pm \gamma_{5} G\right), \quad \bar{P}_{ \pm}=\bar{P}_{ \pm}^{\dagger}=\frac{1}{2}\left(\mathbb{1} \pm \bar{G} \gamma_{5}\right),
$$


where the operators $G(V)$ and $\bar{G}(V)$ satisfy

$$
G^{-1}=G^{\dagger}=\gamma_{5} G \gamma_{5}, \quad \bar{G}^{-1}=\bar{G}^{\dagger}=\gamma_{5} \bar{G} \gamma_{5},
$$

and require that the decomposition

$$
D=\bar{P}_{+} D P_{-}+\bar{P}_{-} D P_{+}
$$

into Weyl operators holds. This immediately leads to the relations

$$
\bar{P}_{ \pm} D P_{\mp}=D P_{\mp}=\bar{P}_{ \pm} D \text {. }
$$

To obtain the condition on $D, G$ and $\bar{G}$ needed in order that (2.19) holds we insert (2.17) into it and find

$$
\bar{G} \gamma_{5} D+D \gamma_{5} G=0 .
$$

Because of the $\gamma_{5}$-Hermiticity of $D, G$ and $\bar{G}$ we can write (2.21) as $\bar{G}^{\dagger} D+D^{\dagger} G=0$, which involves only commuting operators. We thus obtain the condition

$$
D+D^{\dagger} \bar{G} G=0,
$$

which has to be satisfied by $D$. This is seen to generalize our corresponding condition $D+D^{\dagger} V=0$ in Ref. [4, thus still enlarging the class of operators found there.

\subsection{Spectral representations of chiral projections}

With (2.2) $G(V)$ gets the spectral representation

$$
\begin{aligned}
G= & g(1)\left(P_{1}^{(+)}+P_{1}^{(-)}\right)+g(-1)\left(P_{2}^{(+)}+P_{2}^{(-)}\right) \\
& +\sum_{k\left(0<\varphi_{k}<\pi\right)}\left(g\left(\mathrm{e}^{i \varphi_{k}}\right) P_{k}^{(\mathrm{I})}+g\left(\mathrm{e}^{-i \varphi_{k}}\right) P_{k}^{(\mathrm{II})}\right),
\end{aligned}
$$

and $\bar{G}(V)$ the analogous one with the function $g$ replaced by the function $\bar{g}$. According to (2.18) the functions $g$ and $\bar{g}$ satisfy

$$
|g|^{2}=1, \quad g^{*}(v)=g\left(v^{*}\right), \quad|\bar{g}|^{2}=1, \quad \bar{g}^{*}(v)=\bar{g}\left(v^{*}\right) .
$$

In terms of spectral functions condition (2.22) reads

$$
f+f^{*} \bar{g} g=0
$$

For $v=1$ because of (2.12) this is satisfied for any $g$ and $\bar{g}$. However, for $v=-1$ according to (2.13) this leads to the requirement

$$
\bar{g}(-1)=-g(-1)
$$

which causes $\bar{G}$ and $G$ to be generally different. 
For the difference of the numbers $\bar{N}=\operatorname{Tr} \bar{P}_{+}$and $N=\operatorname{Tr} P_{-}$of the Weyl degrees of freedom in $\bar{P}_{+} D P_{-}$, using the spectral representations of $G$ and $\bar{G}$ and condition (2.26), we obtain $\bar{N}-N=\frac{1}{2}(\bar{g}(1)+g(1)) I$. Thus in order to have

$$
\bar{N}-N=I \text {, }
$$

we must put

$$
\bar{g}(1)=g(1)=1
$$

It then similarly follows that

$$
\begin{aligned}
& N=\frac{1}{2} \operatorname{Tr} \mathbb{1}-I, \quad \bar{N}=\frac{1}{2} \operatorname{Tr} \mathbb{1} \quad \text { for } g(-1)=-\bar{g}(-1)=+1, \\
& N=\frac{1}{2} \operatorname{Tr} \mathbb{1}, \quad \bar{N}=\frac{1}{2} \operatorname{Tr} \mathbb{1}+I \quad \text { for } g(-1)=-\bar{g}(-1)=-1 .
\end{aligned}
$$

The spectral representations of $\gamma_{5} G$ and $\bar{G} \gamma_{5}$ now become

$$
\begin{aligned}
& \gamma_{5} G=P_{1}^{(+)}-P_{1}^{(-)}+g(-1)\left(P_{2}^{(+)}-P_{2}^{(-)}\right)+\sum_{k}\left(P_{k}^{[+]}-P_{k}^{[-]}\right), \\
& \bar{G} \gamma_{5}=P_{1}^{(+)}-P_{1}^{(-)}-g(-1)\left(P_{2}^{(+)}-P_{2}^{(-)}\right)+\sum_{k}\left(\bar{P}_{k}^{[+]}-\bar{P}_{k}^{[-]}\right),
\end{aligned}
$$

where the orthogonal projections $P_{k}^{[ \pm]}$and $\bar{P}_{k}^{[ \pm]}$are given by

$$
\begin{aligned}
& P_{k}^{[ \pm]}=\frac{1}{2}\left(1 \pm g\left(\mathrm{e}^{i \varphi_{k}}\right) \gamma_{5}\right) P_{k}^{(\mathrm{I})}+\frac{1}{2}\left(1 \pm g\left(\mathrm{e}^{-i \varphi_{k}}\right) \gamma_{5}\right) P_{k}^{(\mathrm{II})}, \\
& \bar{P}_{k}^{[ \pm]}=P_{k}^{(\mathrm{I})} \frac{1}{2}\left(1 \pm \bar{g}\left(\mathrm{e}^{i \varphi_{k}}\right) \gamma_{5}\right)+P_{k}^{(\mathrm{II})} \frac{1}{2}\left(1 \pm \bar{g}\left(\mathrm{e}^{-i \varphi_{k}}\right) \gamma_{5}\right) .
\end{aligned}
$$

The spectral representations of $P_{-}$and $\bar{P}_{+}$then for $g(-1)=-\bar{g}(-1)= \pm 1$ are

$$
\begin{aligned}
& P_{-}=P_{1}^{(-)}+P_{2}^{(\mp)}+\sum_{k} P_{k}^{[-]}, \\
& \bar{P}_{+}=P_{1}^{(+)}+P_{2}^{(\mp)}+\sum_{k} \bar{P}_{k}^{[+]} .
\end{aligned}
$$

With (2.3) it is obvious that one has $\operatorname{Tr} P_{k}^{[-]}=\operatorname{Tr} \bar{P}_{k}^{[+]}=\operatorname{Tr} P_{k}^{(\mathrm{I})}=\operatorname{Tr} P_{k}^{(\mathrm{II})}=N_{k}$. From (2.32) it is seen that $N-N_{-}(1)=\bar{N}-N_{+}(1)$, reflecting the fact that solely the zero modes of $D$ produce $\bar{N} \neq N$. On the other hand one gets $\tilde{N}:=N-N_{-}(1)=\bar{N}-N_{+}(1)=$ $\sum_{k} N_{k}+N_{\mp}(-1)$ for $g(-1)=-\bar{g}(-1)= \pm 1$, exhibiting the impact of the latter choice on the dimension $N$.

So far (2.22), which in terms of spectral functions is expressed by (2.25), and which is a consequence of $D P_{-}=\bar{P}_{+} D$, has been considered as a condition on $D$. Conversely, for given $D$, it provides a relation between $G$ and $\bar{G}$ and thus between $P_{-}$and $\bar{P}_{+}$. Indeed, for the projections (2.31) in their spectral representations (2.32) with (2.25) and (2.10) one gets explicitly

$$
D P_{k}^{[-]} D^{\dagger}=\left|f\left(\mathrm{e}^{i \varphi_{k}}\right)\right|^{2} \bar{P}_{k}^{[+]}, \quad D^{\dagger} \bar{P}_{k}^{[+]} D=\left|f\left(\mathrm{e}^{i \varphi_{k}}\right)\right|^{2} P_{k}^{[-]} .
$$

Of the other projections in (2.32) $P_{2}^{(\mp)}$ is seen to be related to itself, while for $P_{1}^{(-)}$and $P_{1}^{(+)}$because of $f(1)=0$ by $D$ no relation is provided. 


\section{Realizations of operators}

\subsection{Special cases from literature}

The form implicit in Ref. [1] and given in Ref. [2] in our notation corresponds to choosing

$$
\bar{G}=\mathbb{1}, \quad G=V
$$

with GW operators $V$. This has been extended in Ref. [7] to

$$
\begin{gathered}
G=((1-s) \mathbb{1}+s V) / \mathcal{N}, \quad \bar{G}=(s \mathbb{1}+(1-s) V) / \mathcal{N}, \\
\mathcal{N}=\sqrt{\mathbb{1}-2 s(1-s)\left(\mathbb{1}-\frac{1}{2}\left(V+V^{\dagger}\right)\right)},
\end{gathered}
$$

with a real parameter $s$. With an eigenvalue $\mathrm{e}^{i \varphi}$ of $V$ one gets $1-2 s(1-s)(1-\cos \varphi) \geq 0$ for the respective eigenvalue of $\mathcal{N}^{2}$, which becomes zero for $s=\frac{1}{2}$ and $\varphi=\pi$, so that this definition does not work for $s=\frac{1}{2}$.

The chiral projections used in Ref. [6] in our notation are given by functions $G$ and $\bar{G}$ of the special form (3.2) with the more general operator

$$
V=1-\rho^{-1} D \Psi\left((2 \rho)^{-2} D^{\dagger} D\right),
$$

where $\rho$ is a constant and the operator function $\Psi$ is subject to $\Psi(X)^{\dagger}=\Psi(X)$. The Dirac operators associated to (3.4) have been shown in Ref. [4] to be a special case of the general class there.

For the particular form (3.2) we obtain

$$
\bar{G} G=V .
$$

Therefore according to (2.22) in this case

$$
D+D^{\dagger} V=0
$$

holds, which has been the basic condition on $D$ in Ref. 4]. Thus (3.2) is suitable for the whole class of operators there.

\subsection{Construction of Dirac operator}

Our construction of $D$ in Ref. 4] can be extended to the present more general case. For this purpose we first note that (2.25) can be written as

$$
\left(i(\bar{g} g)^{-\frac{1}{2}} f\right)^{*}=i(\bar{g} g)^{-\frac{1}{2}} f
$$

so that the function $q=i(\bar{g} g)^{-\frac{1}{2}} f$ is real. Therefore $f$ has the form

$$
f\left(\mathrm{e}^{i \varphi}\right)=-i p(\varphi) q(\varphi), \quad q^{*}(\varphi)=q(\varphi), \quad p(\varphi)=\left(\bar{g}\left(\mathrm{e}^{i \varphi}\right) g\left(\mathrm{e}^{i \varphi}\right)\right)^{\frac{1}{2}} .
$$


Noting that with (2.24) we have $\left(p^{2}(\varphi)\right)^{*}=p^{2}(-\varphi)$ we choose the sign such that

$$
p^{*}(\varphi)=p(-\varphi)
$$

With this and (3.8) condition (2.11) gives

$$
q(-\varphi)=-q(\varphi)
$$

so that $q(0)=0$ and $f(1)=0$ hold, as required by (2.12).

Further noting that $p^{2}(\varphi+2 \pi)=p^{2}(\varphi)$ we choose the sign such that

$$
p(\varphi+2 \pi)=-p(\varphi)
$$

which according to (3.8) implies

$$
q(\varphi+2 \pi)=-q(\varphi)
$$

This will allow us to have $q(\pi) \neq 0$ and $f(-1) \neq 0$ as required by (2.13).

With these conditions the spectral function $f$ and thus $D$ can be constructed. They differ from the ones in Ref. [4 only in that instead of the general function $p(\varphi)$, there its special case $\mathrm{e}^{i \varphi / 2}$ occurs. Therefore with respect to the function $q$ we can rely on the result there. Its basic form which satisfies the conditions is $\sin \frac{\varphi}{2}$. This can be multiplied by a real function $w(\cos \varphi)$ provided that $w(-1) \neq 0$ so that (2.13) remains respected. Further, given a function $q$ which satisfies the conditions then also $h(q)$ does if $h$ is a real odd function, which in addition is strictly monotonous so that still (2.13) holds. The steps of multiplying by a function of $\cos \varphi$ and of taking an odd function of the result could be repeated, which we do, however, not consider here. We then have

$$
q(\varphi)=h\left(\sin \frac{\varphi}{2} w(\cos \varphi)\right)
$$

where the real functions of real argument $w$ and $h$ satisfy

$$
\begin{gathered}
w(-1) \neq 0, \\
h(-x)=-h(x), \quad h\left(x_{2}\right)>h\left(x_{1}\right) \text { for } x_{2}>x_{1} .
\end{gathered}
$$

With (3.15) also the inverse function $\eta(y)$ of $h(x)$ is defined and strictly monotonous,

$$
\eta(h(x))=x, \quad \eta(-y)=-\eta(y), \quad \eta\left(y_{2}\right)>\eta\left(y_{1}\right) \text { for } y_{2}>y_{1},
$$

which we shall need in the realization of $V$.

With (3.13) and (3.8) we get the form

$$
f\left(\mathrm{e}^{i \varphi}\right)=-i\left(\bar{g}\left(\mathrm{e}^{i \varphi}\right) g\left(\mathrm{e}^{i \varphi}\right)\right)^{\frac{1}{2}} h\left(\sin \frac{\varphi}{2} w(\cos \varphi)\right),
$$


which inserted into (2.10) gives the Dirac operator $D=F(V)$,

$$
D=-i(\bar{G}(V) G(V))^{\frac{1}{2}} H\left(\frac{1}{2 i}\left(V^{\frac{1}{2}}-V^{-\frac{1}{2}}\right) W\left(\frac{1}{2}\left(V+V^{\dagger}\right)\right)\right),
$$

where the properties of the operator functions $H$ and $W$ correspond to those of the functions $h$ and $w$, respectively, and where the signs of the roots are to be taken as defined in the context of spectral functions.

Several types of concrete examples of (3.18) have been worked out in Ref. [4] and methods to obtain further nontrivial ones have been presented there. Therefore we do not pursue this issue further here.

\subsection{Related form of basic unitary operator}

To specify $V$ explicitly we introduce the normalization-type definition

$$
\begin{gathered}
V=-D_{E}\left(\sqrt{D_{E}^{\dagger} D_{E}}\right)^{-1} \\
D_{E}=-i E\left(\sum_{\mu} \gamma_{\mu} \mathcal{S}_{\mu}\right)+E_{\mathrm{I}}\left(E_{\mathrm{II}}\left(\sum_{\mu}\left(\mathbb{1}-\mathcal{C}_{\mu}\right)\right)-E_{\mathrm{II}}(\vartheta \mathbb{1})\right), \\
\mathcal{S}_{\mu}=\frac{1}{2 i}\left(\mathcal{U}_{\mu}-\mathcal{U}_{\mu}^{\dagger}\right), \quad \mathcal{C}_{\mu}=\frac{1}{2}\left(\mathcal{U}_{\mu}+\mathcal{U}_{\mu}^{\dagger}\right), \quad\left(\mathcal{U}_{\mu}\right)_{n^{\prime} n}=U_{\mu n} \delta_{n^{\prime}, n+\hat{\mu}}^{4},
\end{gathered}
$$

with the gauge-field operator $\mathcal{U}_{\mu}$ and where the properties of the operator function $E$ correspond to those of the real function $\eta$ in (3.16). The functions $E, E_{\mathrm{I}}$ and $E_{\mathrm{II}}$ are Hermitian operator functions of Hermitian argument. They are required to be odd and strictly increasing. This slightly generalizes the respective form in Ref. 4, which arises here putting $E_{\mathrm{I}}=E$ and $E_{\mathrm{II}}(X)=r X$ with $r>0$ and $\vartheta \mathbb{1}=-m / r$. In addition specializing to $E(X)=X$ leads to the Neuberger operator [11]. Instead of (3.19) using the representation 12

$$
V=-D_{E} \frac{1}{\pi} \int_{-\infty}^{\infty} \mathrm{d} s \frac{1}{D_{E}^{\dagger} D_{E}+s^{2}}
$$

one can avoid the square root of noncommuting operators.

To confirm $\gamma_{5}$-Hermiticity of $D_{E}$ one has to consider the individual terms in (3.20). For the function with $\gamma_{\mu}$ there one has to use the spectral representation of its argument for this purpose. Then with $\gamma_{5}$-Hermiticity of $D_{E}$ one gets that of $V$, too.

Having $\gamma_{5}$-Hermiticity of $D_{E}$ one can also introduce the generalized Hermitian WilsonDirac operator $\mathcal{H}=\gamma_{5} D_{E}$, for which one gets

$$
\gamma_{5} V=-\epsilon(\mathcal{H})
$$

providing a further form of the definition of $V$. Since the Hermitian and unitary operator $\gamma_{5} V$ can have only the eigenvalues \pm 1 , according to (3.23) only positive and negative eigenvalues of $\mathcal{H}$ must occur. To exclude zero modes of $\mathcal{H}$ (and thus also of $\mathcal{H}^{2}=D_{E}^{\dagger} D_{E}$ ), 
bounds on the gauge field as in Ref. 22 may be introduced. With (2.16) and (3.23) one gets

$$
I=\frac{1}{2} \operatorname{Tr}\left(\gamma_{5} V\right)=-\frac{1}{2} \operatorname{Tr} \epsilon(\mathcal{H})
$$

showing that the index of $D$ is also given by the difference of the numbers of positive and negative eigenvalues of $\mathcal{H}$. This extends the view of the overlap formalism [1] to the more general operators here.

Checking the continuum limit in the free case for the Fourier transform $\tilde{W}(\cos \varphi)$ of $W$ one gets the condition

$$
\tilde{W}(-1) \neq 0,
$$

with which because of the monotony of $E_{\mathrm{I}}$ and $E_{\mathrm{II}}$ doublers are suppressed for $0<\vartheta<2$. Condition (3.25) corresponds to the requirement $f(-1) \neq 0$ in (2.13), needed to allow for a nonvanishing index. Since we work with dimensionless lattice quantities, in the limit we have $\tilde{D} / a \rightarrow \tilde{D}_{\text {cont }}$. Because of $H(E(X))=X$, putting

$$
\tilde{W}(1)=2|\eta(m)|
$$

the usual normalization of the continuum propagator is obtained.

\section{Transformations of operators}

\subsection{Gauge transformations}

Under gauge transformations the gauge-field operator $\mathcal{U}_{\mu}$ transforms as

$$
\begin{gathered}
\mathcal{U}_{\mu}^{\prime}=\mathcal{T} \mathcal{U}_{\mu} \mathcal{T}^{\dagger}, \quad \mathcal{T}=\mathrm{e}^{\mathcal{B}}, \quad \mathcal{B}^{\dagger}=-\mathcal{B}, \quad\left[\gamma_{5}, \mathcal{B}\right]=0, \\
\left(\mathcal{U}_{\mu}\right)_{n^{\prime} n}=U_{\mu n} \delta_{n^{\prime}, n+\hat{\mu}}^{4}, \quad \mathcal{B}_{n^{\prime} n}=B_{n} \delta_{n^{\prime} n}^{4}, \quad B_{n}=i \sum_{\ell} b_{n}^{\ell} T^{\ell},
\end{gathered}
$$

where $T^{\ell}$ are Hermitian generators and $b_{n}^{\ell}$ is real (and which gives $U_{\mu n}^{\prime}=\mathrm{e}^{B_{n+\hat{\mu}}} U_{\mu n} \mathrm{e}^{-B_{n}}$ ).

Considering the spectral representations of a normal operator $\mathcal{O}$ and the related one of a function $\Phi(\mathcal{O})$ of it,

$$
\mathcal{O}=\sum_{k} \lambda_{k} P_{k}, \quad \Phi(\mathcal{O})=\sum_{k} \phi\left(\lambda_{k}\right) P_{k}
$$

from $\mathcal{O}^{\prime}=\mathcal{T} \mathcal{O} \mathcal{T}^{\dagger}$ we get for the orthogonal projections $P_{k}$ that $P_{k}^{\prime}=\mathcal{T} P_{k} \mathcal{T}^{\dagger}$, which implies that also $\Phi(\mathcal{O})^{\prime}=\mathcal{T} \Phi(\mathcal{O}) \mathcal{T}^{\dagger}$.

According to this the transformations of the operator functions in (3.20) can be traced back to those of their arguments using the individual spectral representations of these arguments. Then with the form (3.22) it is seen that $V$ transforms as

$$
V^{\prime}=\mathcal{T} V \mathcal{T}^{\dagger} .
$$


Further, since the spectral representations (2.10) and (2.23) of $D, G$ and $\bar{G}$ are based on the same projections as (2.2) of $V$, with (4.4) we also have

$$
D^{\prime}=\mathcal{T} D \mathcal{T}^{\dagger}, \quad G^{\prime}=\mathcal{T} G \mathcal{T}^{\dagger}, \quad \bar{G}^{\prime}=\mathcal{T} \bar{G} \mathcal{T}^{\dagger}
$$

With this, (2.17) and $\left[\gamma_{5}, \mathcal{T}\right]=0$ we then further obtain

$$
P_{ \pm}^{\prime}=\mathcal{T} P_{ \pm} \mathcal{T}^{\dagger}, \quad \bar{P}_{ \pm}^{\prime}=\mathcal{T} \bar{P}_{ \pm} \mathcal{T}^{\dagger}
$$

\subsection{CP transformations}

The operators $V, D, G, \bar{G}$ transform under charge conjugation as

$$
\mathcal{O}\left(\mathcal{U}^{\mathrm{C}}\right)=C^{-1}(\mathcal{O}(\mathcal{U}))^{\mathrm{T}} C
$$

where $\mathrm{T}$ denotes transposition in full space, $C$ is the charge conjugation matrix ${ }^{1}$ with $C \gamma_{\mu} C^{-1}=-\gamma_{\mu}^{\mathrm{T}}$ and $C^{\mathrm{T}}=-C$, and where $\mathcal{U}^{\mathrm{C}}=\mathcal{U}^{*}$. To see this we first note that (4.7) is satisfied by the arguments of the operator functions in (3.20). Thus requiring $C^{-1}=C^{\dagger}$ and using the individual spectral representations of these arguments it follows for $D_{E}$, too, and considering (3.22) also for $V$. Then because the spectral representations of $D$, $G$ and $\bar{G}$ are based on that of $V$ this holds also for these operators.

For the parity transformation of the operators $V, D, G, \bar{G}$ we similarly get

$$
\mathcal{O}\left(\mathcal{U}^{\mathrm{P}}\right)=\mathcal{P} \gamma_{4} \mathcal{O}(\mathcal{U}) \gamma_{4} \mathcal{P}
$$

where $\mathcal{P}_{n^{\prime} n}=\delta_{n^{\prime} \tilde{n}}^{4}$ with $\tilde{n}=\left(-\vec{n}, n_{4}\right)$ and where we define $U_{4 n}^{\mathrm{P}}=U_{4 \tilde{n}}$ and $U_{k n}^{\mathrm{P}}=U_{k, \tilde{n}-\hat{k}}$ for $k=1,2,3$.

Combining relations (4.7) and (4.8) we have for the CP transformations of the operators $V, D, G, \bar{G}$

$$
\mathcal{O}\left(\mathcal{U}^{\mathrm{CP}}\right)=\mathcal{W}(\mathcal{O}(\mathcal{U}))^{\mathrm{T}} \mathcal{W}^{\dagger}, \quad \mathcal{W}=\mathcal{P} \gamma_{4} C^{\dagger}
$$

where $\mathcal{W}^{\dagger}=\mathcal{W}^{-1}$.

With (4.9), $\gamma_{5}^{\mathrm{T}}=\gamma_{5}$ and $\left[\gamma_{5}, C\right]=0$ we obtain for the chiral projections $\bar{P}_{+}$and $P_{-}$

$$
\mathcal{W}\left(\bar{P}_{+}(\mathcal{U})\right)^{\mathrm{T}} \mathcal{W}^{\dagger}=P_{-}^{\mathrm{CP}}\left(\mathcal{U}^{\mathrm{CP}}\right), \quad \mathcal{W}\left(P_{-}(\mathcal{U})\right)^{\mathrm{T}} \mathcal{W}^{\dagger}=\bar{P}_{+}^{\mathrm{CP}}\left(\mathcal{U}^{\mathrm{CP}}\right)
$$

where the transformed projections are defined by

$$
P_{-}^{\mathrm{CP}}=\frac{1}{2}\left(\mathbb{1}-\gamma_{5} \bar{G}\right), \quad \bar{P}_{+}^{\mathrm{CP}}=\frac{1}{2}\left(\mathbb{1}+G \gamma_{5}\right) .
$$

This obviously differs from the definitions of $P_{+}$and $\bar{P}_{-}$in (2.17) by an interchange of $G$ and $\bar{G}$. Taking the trace in (4.10) it is seen that one gets $I^{\mathrm{CP}}=-I$ for the index.

\footnotetext{
${ }^{1}$ Using Hermitian $\gamma$-matrices with $\gamma_{\mu}^{\mathrm{T}}=(-1)^{\mu} \gamma_{\mu}$ for $\mu=1, \ldots, 4$ we choose $C=\gamma_{2} \gamma_{4}$. This implies $\gamma_{5}^{\mathrm{T}}=\gamma_{5}$ and $\left[\gamma_{5}, C\right]=0$ for $\gamma_{5}=\gamma_{1} \gamma_{2} \gamma_{3} \gamma_{4}$
} 
A crucial observation now is that, since in (2.22) only the product of $G$ and $\bar{G}$ enters, the same Dirac operator is associated to the operators in (4.11) as to those in (2.17). Applying (4.9) and (4.10) to the Weyl operator $\bar{P}_{+} D P_{-}$we therefore consistently get

$$
\mathcal{W}\left(\bar{P}_{+}(\mathcal{U}) D(\mathcal{U}) P_{-}(\mathcal{U})\right)^{\mathrm{T}} \mathcal{W}^{\dagger}=\bar{P}_{+}^{\mathrm{CP}}\left(\mathcal{U}^{\mathrm{CP}}\right) D\left(\mathcal{U}^{\mathrm{CP}}\right) P_{-}^{\mathrm{CP}}\left(\mathcal{U}^{\mathrm{CP}}\right)
$$

Clearly the interchanged choice of $G$ and $\bar{G}$ produced by the transformation is a legitimate one as well. It is, however, not possible to get the symmetric situation known from the continuum, since here due to (2.26) $\bar{G}$ and $G$ must be generally different.

In more detail from the spectral representations (2.32) it is seen that the two possible choices $g(-1)=-\bar{g}(-1)= \pm 1$ are interchanged under the transformation. In view of the impact of these choices on the dimensions in (2.29), one could think of always fixing one of those dimensions to $\frac{1}{2} \operatorname{Tr} \mathbb{1}$ to avoid a change. With respect to the other terms nothing appears to prevent one from putting $\bar{g}\left(\mathrm{e}^{i \varphi_{k}}\right)=g\left(\mathrm{e}^{-i \varphi_{k}}\right)$ by which one gets $\bar{P}_{k}^{[ \pm]}=P_{k}^{[ \pm]}$and thus no change there.

\section{$5 \quad$ Basis representations of chiral projections}

\subsection{Introduction and transformation of bases}

Noting that in full fermion space the vectors are specified by indices $n, \beta$ and $\alpha$ being related to position space, Dirac space and gauge-group space, respectively, we abbreviate the combination $(n, \alpha, \beta)$ by the index $\sigma$. Basis vectors $u_{j}$ with $j=1, \ldots, N$, which describe the $N$ Weyl degrees of freedom, then can be considered as rectangular matrices of form $u_{\sigma j}$ and rank $N$.

From a more general point of view $u$ provides a mapping from the space $\mathcal{E}_{\mathrm{w}}$ of the Weyl degrees of freedom to the subspace $\mathcal{E}_{P}$ of full fermion space on which $P_{-}$projects, ${ }^{2}$ which both have dimension $N$. The respective transformations back are provided by $u^{\dagger}$ (which outside of $\mathcal{E}_{P}$ maps to zero). Analogous considerations apply to $\bar{P}_{+}$and a related basis $\bar{u}$.

With the indicated understanding basis representations of the chiral projections are introduced by the conditions

$$
P_{-}=u u^{\dagger}, \quad u^{\dagger} u=\mathbb{1}_{\mathrm{w}}, \quad \bar{P}_{+}=\bar{u} \bar{u}^{\dagger}, \quad \bar{u}^{\dagger} \bar{u}=\mathbb{1}_{\overline{\mathrm{w}}},
$$

where $\mathbb{1}_{\mathrm{w}}$ and $\mathbb{1}_{\overline{\mathrm{w}}}$ are the identity operators in the spaces of the degrees of freedom of Weyl fermions $\mathcal{E}_{\mathrm{w}}$ and of Weyl anti-fermions $\mathcal{E}_{\overline{\mathrm{w}}}$, repectively.

While the choice of the bases is not unique, different ones of them must represent the same projection. Thus they are related by unitary transformations,

$$
u^{(S)}=u S, \quad S^{-1}=S^{\dagger}, \quad \bar{u}^{(\bar{S})}=\bar{u} \bar{S}, \quad \bar{S}^{-1}=\bar{S}^{\dagger},
$$

so that one generally gets $P_{-}=u^{(S)} u^{(S) \dagger}$ and $\bar{P}_{+}=\bar{u}^{(\bar{S})} \bar{u}^{(\bar{S}) \dagger}$. Obviously $S$ and $\bar{S}$ operate within $\mathcal{E}_{\mathrm{w}}$ and $\mathcal{E}_{\overline{\mathrm{w}}}$, respectively.

\footnotetext{
${ }^{2}$ Between $\mathcal{E}_{P}$ and $\mathcal{E}_{\mathrm{w}}$ the mapping is even unitary, see e.g. Ref. 13 for the definition of unitary operators acting between different spaces.
} 


\subsection{Gauge transformations}

According to $P_{-}^{\prime}=\mathcal{T} P_{-} \mathcal{T}^{\dagger}$ from (4.6), given a solution $u$ which satisfies conditions $P_{-}=u u^{\dagger}$ and $u^{\dagger} u=\mathbb{1}_{\mathrm{w}}$ in (5.1), then $\mathcal{T} u$ is a solution of the transformed conditions, $P_{-}^{\prime}=u^{\prime} u^{\prime \dagger}$ and $u^{\prime \dagger} u^{\prime}=\mathbb{1}_{\mathrm{w}}$. Furthermore, then also $\mathcal{T} u S$ with any $S$ from (5.2) is a solution of the latter, and inserting all possible $S$ one gets all such solutions. Analogous conclusions hold for $\bar{u}$, so that we generally have the forms

$$
u^{\prime}=\mathcal{T} u S, \quad \bar{u}^{\prime}=\mathcal{T} \bar{u} \bar{S} .
$$

The cases $G=\mathbb{1}, \bar{G} \neq \mathbb{1}$ and $G \neq \mathbb{1}, \bar{G}=\mathbb{1}$ are exceptional in that $\mathcal{T}$ commutes with $P_{-}$and $\bar{P}_{+}$, respectively, so that the gauge-field dependences of the respective bases are no longer restricted. To see for $\bar{G}=\mathbb{1}$ how this gets consistent with (5.3) one notes that one can put

$$
\mathcal{T} \bar{u}=\bar{u} \bar{S}_{\mathcal{T}} \quad \text { for } \quad\left[\mathcal{T}, \bar{P}_{+}\right]=0,
$$

which allows to trade $\mathcal{T}$ for the basis transformation with

$$
\bar{S}_{\mathcal{T}}=\bar{u}^{\dagger} \mathcal{T} \bar{u} .
$$

In this way $\mathcal{T} \bar{P}_{+} \mathcal{T}^{\dagger}=\bar{P}_{+}$is realized within $\bar{P}_{+}=\bar{u} \bar{u}^{\dagger}$. The particular case $\bar{u}^{\prime}=\bar{u}$ is seen to arise by choosing $\bar{S}=\bar{S}_{\mathcal{T}}^{\dagger}$ in (5.3).

In the case $G \neq \mathbb{1}, \bar{G} \neq \mathbb{1}$, where no trading (5.4) is possible, (5.3) can be considered as a combination of a basis transformation (5.2) and the gauge transformation

$$
u^{\prime}=\mathcal{T} u, \quad \bar{u}^{\prime}=\mathcal{T} \bar{u} \quad \text { for } \quad G \neq \mathbb{1}, \quad \bar{G} \neq \mathbb{1} .
$$

In the exceptional case $G \neq \mathbb{1}, \bar{G}=\mathbb{1}$ for the bases $\bar{u}$ one can start from a basis $\bar{u}_{\mathrm{c}}$ which is independent of the gauge field and gets the other ones by basis transformations $\bar{u}=\bar{u}_{\mathrm{c}} \bar{S}$. Then instead of (5.6) one has

$$
u^{\prime}=\mathcal{T} u, \quad \bar{u}_{\mathrm{c}}^{\prime}=\bar{u}_{\mathrm{c}} \quad \text { for } \quad G \neq \mathbb{1}, \quad \bar{G}=\mathbb{1} .
$$

Combining basis transformations with the transformations (5.6) and (5.7) all possible bases are reached, with the important consequence that actually the whole original set of bases is related to the whole transformed one. A simple equivalent view of this is that in the transformation laws (5.6) and (5.7) each basis can be any one of the respective set.

\subsection{CP transformations}

Given a solution $u$ of the conditions (5.1), then according to (4.10) $\mathcal{W} \bar{u}^{*}$ is a solution of the CP transformed conditions. With analogous conclusions for $\bar{u}$ we thus arrive (with the dependences $\left.u(\mathcal{U}), \bar{u}(\mathcal{U}), u^{\mathrm{CP}}\left(\mathcal{U}^{\mathrm{CP}}\right), \bar{u}^{\mathrm{CP}}\left(\mathcal{U}^{\mathrm{CP}}\right)\right)$ at

$$
u^{\mathrm{CP}}=\mathcal{W} \bar{u}^{*}, \quad \bar{u}^{\mathrm{CP}}=\mathcal{W} u^{*},
$$


solving the transformed conditions

$$
\bar{P}_{+}^{\mathrm{CP}}=\bar{u}^{\mathrm{CP}} \bar{u}^{\mathrm{CP} \dagger}, \quad \bar{u}^{\mathrm{CP} \dagger} \bar{u}^{\mathrm{CP}}=\mathbb{1}_{\mathrm{w}}, \quad P_{-}^{\mathrm{CP}}=u^{\mathrm{CP}} u^{\mathrm{CP} \dagger}, \quad u^{\mathrm{CP} \dagger} u^{\mathrm{CP}}=\mathbb{1}_{\overline{\mathrm{w}}}
$$

(in which the interchange of $G$ and $\bar{G}$ in (4.11) as compared to (2.17) is implicit). Combining the transformations (5.8) with basis transformations all possible bases are reached, so that actually the whole original set of bases is related to the whole transformed one. An equivalent view is that in the transformation law (5.8) each basis can be any one of the respective set.

\section{Correlation Functions}

\subsection{Definitions and general relations}

Associating Grassmann variables $\chi_{k}$ and $\bar{\chi}_{j}$ to the $N$ degrees of freedom of left-handed fermions and the $\bar{N}$ ones of right-handed anti-fermions, respectively, the fermion field variables $\psi_{\sigma}$ and $\bar{\psi}_{\sigma^{\prime}}$ get

$$
\bar{\psi}=\bar{\chi} \bar{u}^{\dagger}, \quad \psi=u \chi .
$$

The fermion action then is given by

$$
S_{\mathrm{f}}=\bar{\psi} D \psi=\bar{\chi} M \chi
$$

where the matrix $M$, which maps from $\mathcal{E}_{\mathrm{w}}$ to $\mathcal{E}_{\overline{\mathrm{w}}}$, is

$$
M=\bar{u}^{\dagger} D u \text {. }
$$

For a given value of the index $I$ the numbers $N$ and $\bar{N}$ are already both determined since according to (2.29) either $N$ or $\bar{N}$ gets the fixed value $\frac{1}{2} \operatorname{Tr} \mathbb{1}$ and with (2.27) one generally has $\bar{N}-N=I$. Fermionic correlation functions $\left\langle\chi_{i_{1}} \ldots \chi_{i_{L}} \bar{\chi}_{j_{1}} \ldots \bar{\chi}_{j_{\bar{L}}}\right\rangle_{\mathrm{f}}$ then can be nonvanishing only for

$$
L-N=\bar{L}-\bar{N}=r, \quad 0 \leq r \leq \min (\bar{N}, N), \quad \bar{L}-L=\bar{N}-N=I,
$$

where $\min (\bar{N}, N)=\frac{1}{2}(g(-1) I-|I|)$. We define such nonvanishing functions by

$$
\begin{aligned}
\left\langle\chi_{i_{r+1}}\right. & \left.\ldots \chi_{i_{N}} \bar{\chi}_{j_{r+1}} \ldots \bar{\chi}_{j_{\bar{N}}}\right\rangle_{\mathrm{f}} \\
& =s_{r} \int \mathrm{d} \bar{\chi}_{\bar{N}} \ldots \mathrm{d} \bar{\chi}_{1} \mathrm{~d} \chi_{N} \ldots \mathrm{d} \chi_{1} \mathrm{e}^{-S_{\mathrm{f}}} \chi_{i_{r+1}} \ldots \chi_{i_{N}} \bar{\chi}_{j_{r+1}} \ldots \bar{\chi}_{\bar{j}_{\bar{N}}} \\
& =\frac{1}{r !} \sum_{j_{1}, \ldots, j_{r}=1}^{N} \sum_{i_{1}, \ldots, i_{r}=1}^{N} \epsilon_{j_{1}, \ldots, j_{\bar{N}}} \epsilon_{i_{1}, \ldots, i_{N}} M_{j_{1} i_{1}} \ldots, M_{j_{r} i_{r}},
\end{aligned}
$$

where $s_{r}$ is the sign factor $s_{r}=(-1)^{r N-r(r+1) / 2}$. 
With (6.5) using (6.1) we get for the fields $\psi$ and $\bar{\psi}$

$$
\begin{aligned}
& \left\langle\psi_{\sigma_{r+1}} \ldots \psi_{\sigma_{N}} \bar{\psi}_{\bar{\sigma}_{r+1}} \ldots \bar{\psi}_{\bar{\sigma}_{\bar{N}}}\right\rangle_{\mathrm{f}} \\
& =s_{r} \int \mathrm{d} \bar{\chi}_{\bar{N}} \ldots \mathrm{d} \bar{\chi}_{1} \mathrm{~d} \chi_{N} \ldots \mathrm{d} \chi_{1} \mathrm{e}^{-S_{\mathrm{f}}} \psi_{\sigma_{r+1}} \ldots \psi_{\sigma_{N}} \bar{\psi}_{\bar{\sigma}_{r+1}} \ldots \bar{\psi}_{\bar{\sigma}_{\bar{N}}} \\
& =\frac{1}{r !} \sum_{j_{1}, \ldots, j_{\bar{N}}=1}^{N} \sum_{i_{1}, \ldots, i_{N}=1}^{N} \epsilon_{j_{1}, \ldots, j_{\bar{N}}} \epsilon_{i_{1}, \ldots, i_{N}} M_{j_{1} i_{1}} \ldots M_{j_{r} i_{r}} \\
& \bar{u}_{j_{r+1} \bar{\sigma}_{r+1}}^{\dagger} \ldots \bar{u}_{j_{\bar{N}} \bar{\sigma}_{\bar{N}}}^{\dagger} u_{\sigma_{r+1} i_{r+1}} \ldots u_{\sigma_{N} i_{N}} .
\end{aligned}
$$

In Ref. 2 the question of different complex factors multiplying the fermionic correlation functions for different values $I$ has been raised. There is, however, no theoretical principle describing this. An explicit reason for these factors could be the $I$-dependence in (2.29). In Ref. [7] the importance of such factors for the magnitude of fermion number violating processes has been stressed. In Refs. [16, 6] suggestions that the modulus of them could possibly be generally one have been made. The alternating-form representations to be introduced in Section 7 might even suggest that there are no such factors.

With the question of the indicated factors somehow settled, more general fermionic correlation functions can readily be constructed as linear combinations of the functions we have introduced. The inclusion of the gauge fields and the definition of full correlation functions then is straightforward and needs not to be considered here.

\subsection{Basis transformations}

Requiring that the field variables $\psi$ and $\bar{\psi}$ are not affected by the basis transformations (5.2) induces transformations of the Grassmann variables $\chi$ and $\bar{\chi}$, too,

$$
\chi^{\left(S^{\dagger}\right)}=S^{\dagger} \chi, \quad \bar{\chi}^{(\bar{S})}=\bar{\chi} \bar{S} .
$$

This has the consequence that the fermionic integration measure transforms as

$$
\mathrm{d} \bar{\chi}_{\bar{N}}^{(\bar{S})} \ldots \mathrm{d} \bar{\chi}_{1}^{(\bar{S})} \mathrm{d} \chi_{N}^{\left(S^{\dagger}\right)} \ldots \mathrm{d} \chi_{1}^{\left(S^{\dagger}\right)}=\operatorname{det}_{\overline{\mathrm{w}}} \bar{S} \operatorname{det}_{\mathrm{w}} S^{\dagger} \mathrm{d} \bar{\chi}_{\bar{N}} \ldots \mathrm{d} \bar{\chi}_{1} \mathrm{~d} \chi_{N} \ldots \mathrm{d} \chi_{1}
$$

where $\operatorname{det}_{\overline{\mathrm{w}}}$ and $\operatorname{det}_{\mathrm{w}}$ denote the determinants in the spaces $\mathcal{E}_{\overline{\mathrm{w}}}$ and $\mathcal{E}_{\mathrm{w}}$, respectively. Thus, in order to get invariance of the correlation functions (6.6) we have to impose the additional conditions

$$
\operatorname{det}_{\mathrm{w}} S=1, \quad \operatorname{det}_{\overline{\mathrm{w}}} \bar{S}=1,
$$

i.e. to restrict the basis transformations to unimodular ones. ${ }^{3}$

Conditions (6.9) have important consequences for the possible sets of bases. In the case of $u$ (analogous considerations apply to $\bar{u}$ ) without the restriction (6.9) the unitary

\footnotetext{
${ }^{3}$ For general expectations different constant phase factors of their contributions would lead to different interference terms in the moduli of the amplitudes. In order that basis transformations in different contributions cannot cause such an effect the determinants must be fixed to a universal constant, which without restricting generality can be choosen to be one.
} 
transformations $S$ connect all bases of the unitary space $\mathcal{E}_{P}$ on which $P_{-}$projects. The unimodular $S$ only connect subsets of the total set of these bases, so that the total set is decomposed into subsets. Our formulation of the theory thus has to be restricted to one of such subsets. The task then is to choose the appropriate one of them.

We note that starting from an arbitrary basis the particular spectral decompositions of the chiral projections in (2.32) can also be reached by unimodular transformations. This is so because a unitary transformation can be expressed as a product of a unimodular transformation and of the identity operator times a phase factor. Thus within this respect no restrictions arise.

Our discusssions of transformation properties, so far given for the total set, apply as well to the subsets of bases. Some more detailed considerations (see Subsection 6.3) are only needed in the exceptional cases for gauge transformations. Generally the rule is that the symmetries of the chiral projections give that of the bases, where the latter are only fixed up to unimodular basis transformations.

The remaining problem then is that formulations in different subsets are not equivalent. The non-unimodular basis transformations, which transform between inequivalent subsets, produce phase factors. Such a phase factor describes how the results of the formulation of the theory in one subset differ from those of the formulation of the theory in another subset which is inequivalent to the former one. Obviously a criterion is needed, telling which one of such subsets is describing physics.

\subsection{Gauge transformations}

So far the combinations of the gauge transformations (5.6) and (5.7) with basis transformations (5.2) have been recognized to constitute the gauge transformations of the whole set of bases to the whole transformed set. After imposing conditions (6.9) the combinations of the unimodular basis transformations with the gauge transformations (15.6) and (5.7) give the transformations of the whole subset to the whole transformed subset. In this context it is to be noted that in the exceptional cases the subset of bases related to the gauge-field independent chiral projection necessarily contains a gauge-field independent basis.

With the correlation functions being invariant under unimodular basis transformations, in the non-exceptional case it suffices to use (5.6) to get the transformation properties. Accordingly inserting (5.6) into (6.6), the correlation functions are seen to transform as

$$
\begin{aligned}
& \left\langle\psi_{\sigma_{1}^{\prime}}^{\prime} \ldots \psi_{\sigma_{L}^{\prime}}^{\prime} \bar{\psi}_{\bar{\sigma}_{1}^{\prime}}^{\prime} \ldots \bar{\psi}_{\bar{\sigma}_{\bar{L}}^{\prime}}^{\prime}\right\rangle_{\mathrm{f}}^{\prime} \\
& \quad=\sum_{\sigma_{1}, \ldots, \sigma_{L}} \sum_{\bar{\sigma}_{1}, \ldots, \bar{\sigma}_{\bar{L}}} \mathcal{T}_{\sigma_{1}^{\prime} \sigma_{1}} \ldots \mathcal{T}_{\sigma_{L}^{\prime} \sigma_{L}}\left\langle\psi_{\sigma_{1}} \ldots \psi_{\sigma_{L}} \bar{\psi}_{\bar{\sigma}_{1}} \ldots \bar{\psi}_{\bar{\sigma}_{\bar{L}}}\right\rangle_{\mathrm{f}} \mathcal{T}_{\bar{\sigma}_{1} \bar{\sigma}_{1}^{\prime}}^{\dagger} \ldots \mathcal{T}_{\bar{\sigma}_{\bar{L}} \bar{\sigma}_{\bar{L}}^{\prime}}^{\dagger} \\
& \text { for } \quad G \neq \mathbb{1}, \quad \bar{G} \neq \mathbb{1} .
\end{aligned}
$$

In the exceptional case $G \neq \mathbb{1}, \bar{G}=\mathbb{1}$ with the transformation (5.7) we can apply the trading (5.4) to $\bar{u}_{\mathrm{c}}$ to get $\bar{u}_{\mathrm{c}}=\mathcal{T} \bar{u}_{\mathrm{c}} \bar{S}_{\mathcal{T}}^{\dagger}$ (where, of course, (6.9) needs not to hold for 
$\bar{S}_{\mathcal{T}}$ ). With this the form (6.10) is seen to be supplemented by the constant phase factor $\operatorname{det}_{\overline{\mathrm{w}}} \bar{S}_{\mathcal{T}}=\operatorname{det}_{\overline{\mathrm{w}}}\left(\bar{u}_{\mathrm{c}}^{\dagger} \mathcal{T} \bar{u}_{\mathrm{c}}\right)$. To calculate this factor we represent the determinant by 14

$$
\operatorname{det}_{\overline{\mathrm{w}}} \bar{S}=(-1)^{\bar{N}} \sum_{r=1}^{\bar{N}} \frac{(-1)^{r}}{r !} \sum_{\rho_{1}=1}^{\bar{N}-r+1} \ldots \sum_{\rho_{r}=1}^{\bar{N}-r+1} \delta_{\bar{N}, \rho_{1}+\ldots+\rho_{r}} \frac{\operatorname{Tr}_{\overline{\mathrm{w}}}\left(\bar{S}^{\rho_{1}}\right)}{\rho_{1}} \ldots \frac{\operatorname{Tr}_{\overline{\mathrm{w}}}\left(\bar{S}^{\rho_{r}}\right)}{\rho_{r}}
$$

and note that with $\left[\mathcal{T}, \bar{P}_{+}\right]=0$ and $\mathcal{T}=e^{\mathcal{B}}$ we have

$$
\operatorname{Tr}_{\overline{\mathrm{w}}}\left(\bar{S}^{\rho}\right)=\operatorname{Tr}_{\overline{\mathrm{w}}}\left(\left(\bar{u}_{c}^{\dagger} \mathcal{T} \bar{u}_{c}\right)^{\rho}\right)=\operatorname{Tr}\left(\left(\bar{P}_{+} \mathcal{T}\right)^{\rho}\right)=\operatorname{Tr}\left(\bar{P}_{+} \mathrm{e}^{\rho \mathcal{B} \bar{P}_{+}}\right) .
$$

Since $\mathcal{E}_{\overline{\mathrm{w}}}$ and $\mathcal{E}_{\bar{P}}$ both have dimension $\bar{N}$, we can use (6.12) to replace $\operatorname{Tr}_{\overline{\mathrm{w}}}\left(\bar{S}^{\rho}\right)$ in (6.11) and with $\bar{P}_{+}=\frac{1}{2}\left(1+\gamma_{5}\right) \mathbb{1}$ get

$$
\operatorname{det}_{\overline{\mathrm{w}}} \bar{S}=\operatorname{det}_{\bar{P}}\left(\mathrm{e}^{\mathcal{B} \bar{P}_{+}}\right)=\mathrm{e}^{\operatorname{Tr}\left(\mathcal{B} \bar{P}_{+}\right)}=\mathrm{e}^{\frac{1}{2} \operatorname{Tr} \mathcal{B}} .
$$

Analogously for $G=\mathbb{1}, \bar{G} \neq \mathbb{1}$, (6.10) is to be multiplied by the the phase factor $\mathrm{e}^{-\frac{1}{2} \operatorname{Tr} \mathcal{B}}$. Because with (4.2) we have in more detail $\frac{1}{2} \operatorname{Tr} \mathcal{B}=2 i \sum_{n, \ell} b_{n}^{\ell} \operatorname{tr}_{\mathrm{g}} T^{\ell}$, where the $\operatorname{trace} \operatorname{tr}_{\mathrm{g}}$ applies to gauge-field space only, obviously the additional condition $\operatorname{tr}_{\mathrm{g}} T^{\ell}=0$ is needed to get rid of these extra factors. ${ }^{4}$

\subsection{CP transformations}

The combination of the CP transformations (5.8) with basis transformations (5.2) that satisfy in addition (6.9) constitutes the CP transformation of the whole respective subset of bases to the whole transformed subset. Since the correlation functions are invariant under such basis transformations, it suffices to use (5.8) to derive their CP-transformation properties.

Inserting (4.9) and (5.8) we get for the matrix (6.3)

$$
M^{\mathrm{CP}}\left(\mathcal{U}^{\mathrm{CP}}\right)=M^{\mathrm{T}}(\mathcal{U}) .
$$

With (6.14) and (5.8) we obtain for the transformed form of (6.6)

$$
\begin{aligned}
& \frac{1}{r !} \sum_{j_{1}, \ldots, j_{\bar{N}}=1}^{\bar{N}} \sum_{i_{1}, \ldots, i_{N}=1}^{N} \epsilon_{j_{1}, \ldots, j_{\bar{N}}} \epsilon_{i_{1}, \ldots, i_{N}} M_{j_{1} i_{1}}^{\mathrm{CP}} \ldots M_{j_{r} i_{r}}^{\mathrm{CP}} \\
& \bar{u}_{j_{r+1} \bar{\sigma}_{r+1}}^{\mathrm{CP} \dagger} \ldots \bar{u}_{j_{\bar{N}} \overline{\sigma_{\bar{N}}}}^{\mathrm{CP} \dagger} u_{\sigma_{r+1} i_{r+1}}^{\mathrm{CP}} \ldots u_{\sigma_{N} i_{N}}^{\mathrm{CP}} \\
& =\frac{1}{r !} \sum_{j_{1}, \ldots, j_{\bar{N}}=1}^{\bar{N}} \sum_{i_{1}, \ldots, i_{N}=1}^{N} \sum_{\bar{\sigma}_{r+1}^{\prime}, \ldots \bar{\sigma}_{\bar{N}}^{\prime} \sigma_{r+1}^{\prime}, \ldots \sigma_{N}^{\prime}} \sum_{j_{1}, \ldots, j_{\bar{N}} \epsilon_{i_{1}, \ldots, i_{N}}} M_{i_{1} j_{1}} \ldots M_{i_{r} j_{r}} \\
& \mathcal{W}_{\bar{\sigma}_{r+1}^{\prime} \bar{\sigma}_{r+1}}^{\dagger} \ldots \mathcal{W}_{\bar{\sigma}_{N}^{\prime} \bar{\sigma}_{\bar{N}}}^{\dagger} u_{\bar{\sigma}_{r+1}^{\prime} j_{r+1}} \ldots u_{\bar{\sigma}_{\bar{N}}^{\prime} j_{\bar{N}}} \bar{u}_{i_{r+1} \sigma_{r+1}^{\prime}}^{\dagger} \ldots \bar{u}_{i_{N} \sigma_{N}^{\prime}}^{\dagger} \mathcal{W}_{\sigma_{r+1} \sigma_{r+1}^{\prime}} \ldots \mathcal{W}_{\sigma_{N} \sigma_{N}^{\prime}} \text {. }
\end{aligned}
$$

\footnotetext{
${ }^{4}$ Since these factors can be different in different fermionic contributions, similarly as the constant ones discussed in the context of basis transformation, they are required to be equal to one. The condition for this, $\operatorname{tr}_{\mathrm{g}} T^{\ell}=0$, holds in the Standard Model.
} 
This shows that the correlation functions (6.6) transform as

$$
\begin{aligned}
& \left\langle\psi_{\sigma_{1}^{\prime}}^{\mathrm{CP}} \ldots \psi_{\sigma_{L}^{\prime}}^{\mathrm{CP}} \bar{\psi}_{\bar{\sigma}_{1}^{\prime}}^{\mathrm{CP}} \ldots \bar{\psi}_{\bar{\sigma}_{\bar{L}}^{\prime}}^{\mathrm{CP}}\right\rangle_{\mathrm{f}}^{\mathrm{CP}} \\
& =\sum_{\sigma_{1}, \ldots, \sigma_{L}} \sum_{\bar{\sigma}_{1}, \ldots, \bar{\sigma}_{\bar{L}}} \mathcal{W}_{\bar{\sigma}_{1} \bar{\sigma}_{1}^{\prime}}^{\dagger} \ldots \mathcal{W}_{\bar{\sigma}_{\bar{L}^{\prime}} \bar{\sigma}_{\bar{L}}^{\prime}}^{\dagger}\left\langle\psi_{\bar{\sigma}_{1}} \ldots \psi_{\bar{\sigma}_{\bar{L}}} \bar{\psi}_{\sigma_{1}} \ldots \bar{\psi}_{\sigma_{L}}\right\rangle_{\mathrm{f}} \mathcal{W}_{\sigma_{1}^{\prime} \sigma_{1}} \ldots \mathcal{W}_{\sigma_{L}^{\prime} \sigma_{L}} .
\end{aligned}
$$

It is to be remembered here that according to (4.11) an interchange of $G$ and $\bar{G}$ is inherent in this, which in Subsection 4.2 has been discussed in detail.

\subsection{Case of index zero and chiral determinant}

In the special case $\bar{L}=L$, where one gets a nonvanishing function for $\bar{N}=N$ and $I=0$ only, (6.6) can also be written in the form ${ }^{5}$

$$
\begin{aligned}
& \left\langle\psi_{\sigma_{r+1}} \ldots \psi_{\sigma_{N}} \bar{\psi}_{\bar{\sigma}_{r+1}} \ldots \bar{\psi}_{\bar{\sigma}_{N}}\right\rangle_{\mathrm{f}}=\int \prod_{l=1}^{N}\left(\mathrm{~d} \bar{\chi}_{l} \mathrm{~d} \chi_{l}\right) \mathrm{e}^{-S_{\mathrm{f}}} \psi_{\sigma_{r+1}} \bar{\psi}_{\bar{\sigma}_{r+1}} \ldots \psi_{\sigma_{N}} \bar{\psi}_{\bar{\sigma}_{N}}= \\
& \sum_{\sigma_{r+1}^{\prime}, \ldots, \sigma_{N}^{\prime}} \epsilon_{\sigma_{r+1} \ldots \sigma_{N}}^{\sigma_{r+1}^{\prime} \ldots \sigma_{N}^{\prime}}\left(P_{-} D^{-1} \bar{P}_{+}\right)_{\sigma_{r+1}^{\prime} \bar{\sigma}_{r+1}} \ldots\left(P_{-} D^{-1} \bar{P}_{+}\right)_{\sigma_{N}^{\prime} \bar{\sigma}_{N}} \operatorname{det}_{\overline{\mathrm{w}} \mathrm{w}} M
\end{aligned}
$$

where the notation $\operatorname{det}_{\overline{\mathrm{w}} \mathrm{w}}$ indicates that the determinant here actually involves a matrix connecting the different spaces $\mathcal{E}_{\mathrm{w}}$ and $\mathcal{E}_{\overline{\mathrm{w}}}$. Correspondingly for $M^{-1}$ one has the slightly generalized defining relations $M^{-1} M=\mathbb{1}_{\mathrm{w}}$ and $M M^{-1}=\mathbb{1}_{\overline{\mathrm{w}}}$.

While in the form (6.6) the presence of zero modes of $D$ is no problem, in (6.17) one needs to care about them. If $M^{-1}$ exists, using (2.10) it follows from $M^{-1} M=\mathbb{1}_{\mathrm{w}}$ that $P_{1}^{(-)}=0$ and from $M M^{-1}=\mathbb{1}_{\overline{\mathrm{w}}}$ that $P_{1}^{(+)}=0$, so that $P_{1}^{(+)}+P_{1}^{(-)}$vanishes and $D^{-1}$ exists, too. Conversely, if $D^{-1}$ exists, putting $M^{-1}=u^{\dagger} D^{-1} \bar{u}$ it is seen that $M^{-1}$ exists, too. Thus, if there are zero modes of $D$ it follows that $M$ is not invertible, which implies $\operatorname{det}_{\overline{\mathrm{w}} \mathrm{w}} M=0$ and also that $P_{-} D^{-1} \bar{P}_{+}=u M^{-1} \bar{u}$ does not exist.

The basis independence of (6.17) becomes obvious noting that with (6.9) one gets $\operatorname{det}_{\overline{\mathrm{w}} \mathrm{w}}\left(\bar{S}^{\dagger} M S\right)=\left(\operatorname{det}_{\overline{\mathrm{w}}} \bar{S}^{\dagger}\right)\left(\operatorname{det}_{\overline{\mathrm{w}} \mathrm{w}} M\right) \operatorname{det}_{\mathrm{w}} S=\operatorname{det}_{\overline{\mathrm{w} w}} M$. The chiral determinant from (6.10) is seen to be gauge invariant for $\bar{G} \neq \mathbb{1}, G \neq \mathbb{1}$, while in the exceptional cases $G \neq \mathbb{1}$, $\bar{G}=\mathbb{1}$ and $G=\mathbb{1}, \bar{G} \neq \mathbb{1}$ the extra factors $\mathrm{e}^{\frac{1}{2} \operatorname{Tr} \mathcal{B}}$ and $\mathrm{e}^{-\frac{1}{2} \operatorname{Tr} \mathcal{B}}$, respectively, occur. Under CP transformations because of (6.14) one has $\operatorname{det}_{\mathrm{w} \overline{\mathrm{w}}} M^{\mathrm{CP}}\left(\mathcal{U}^{\mathrm{CP}}\right)=\operatorname{det}_{\overline{\mathrm{w}} \mathrm{w}} M(\mathcal{U})$.

\subsection{Effective action}

To evaluate the chiral determinant we write it as 14

$$
\operatorname{det}_{\overline{\mathrm{w}} \mathrm{w}} M=(-1)^{N} \sum_{r=1}^{N} \frac{(-1)^{r}}{r !} \sum_{\rho_{1}=1}^{N-r+1} \ldots \sum_{\rho_{r}=1}^{N-r+1} \delta_{N, \rho_{1}+\ldots+\rho_{r}} \frac{\operatorname{Tr}_{\overline{\mathrm{w}} \mathrm{w}}\left(M^{\rho_{1}}\right)}{\rho_{1}} \ldots \frac{\operatorname{Tr}_{\overline{\mathrm{w}} \mathrm{w}}\left(M^{\rho_{r}}\right)}{\rho_{r}}
$$

${ }^{5}$ Note that $\epsilon_{j_{1}, \ldots, j_{r}}^{i_{1}, \ldots, i_{r}}=1,-1$ or 0 if $i_{1}, \ldots, i_{r}$ is an even, an odd or no permutation of $j_{1}, \ldots, j_{r}$, respectively, with the special case $\epsilon_{j_{1}, \ldots, j_{N}} \equiv \epsilon_{j_{1}, \ldots, j_{N}}^{1, \ldots, N}$. 
and note that putting $Q=u \bar{u}^{\dagger}$ we have

$$
\operatorname{Tr}_{\overline{\mathrm{w}} \mathrm{W}}\left(M^{\rho}\right)=\operatorname{Tr}\left((Q D)^{\rho}\right)=\operatorname{Tr}\left(P_{-}(Q D)^{\rho} P_{-}\right)=\operatorname{Tr}\left(\bar{P}_{+}(D Q)^{\rho} \bar{P}_{+}\right) .
$$

Since $\mathcal{E}_{\overline{\mathrm{w}}}, \mathcal{E}_{\mathrm{w}}, \mathcal{E}_{\bar{P}}$ and $\mathcal{E}_{P}$ here all have dimension $N$, we can use (6.19) to replace $\operatorname{Tr}_{\overline{\mathrm{ww}}}\left(M^{\rho}\right)$ in (6.18) and obtain the factorization

$$
\operatorname{det}_{\overline{\mathrm{w}} \mathrm{W}} M=\operatorname{det}_{P}(Q D)=\operatorname{det}_{\bar{P}}(D Q)=\operatorname{det}_{P \bar{P}}(Q) \operatorname{det}_{\bar{P} P}(D),
$$

where $\operatorname{det}_{\bar{P} P}(D)$ is the contribution of the Weyl operator $\bar{P}_{+} D P_{-}$while $\operatorname{det}_{P \bar{P}}(Q)$ is the contribution of the bases. For the effective action we then have

$$
\ln \operatorname{det}_{\overline{\mathrm{w}} \mathrm{w}} M=\operatorname{Tr} \ln Q+\operatorname{Tr} \ln \left(\bar{P}_{+} D P_{-}\right) .
$$

The locality of the Weyl operator $\bar{P}_{+} D P_{-}$in (6.21) relies on that of $D, P_{-}$and $\bar{P}_{+}$, which inherit locality from $V$. To study this the spectral representation of $V$ and the related ones of $F(V), G(V)$ and $\bar{G}(V)$ can be used. Considering the continuum analogon $V(x, y)=\sum_{k} v_{k} \phi_{k}(x) \phi_{k}^{\dagger}(y)$ for this we have, for example, $D(x, y)=\sum_{k} f\left(v_{k}\right) \phi_{k}(x) \phi_{k}^{\dagger}(y)$. Then considering the decrease of $\phi_{k}(y)$ and the orthogonality of the individual terms we see how the locality transfers.

For local $P_{-}=u u^{\dagger}$ and $\bar{P}_{+}=\bar{u} \bar{u}^{\dagger}$ the operator $Q=u \bar{u}^{\dagger}$ in (6.21) gets local, too. To see this we consider the continuum analogues $P(x, y)=\sum_{k} u_{k}(x) u_{k}^{\dagger}(y), \bar{P}(x, y)=$ $\sum_{k} \bar{u}_{k}(x) \bar{u}_{k}^{\dagger}(y)$ and $Q(x, y)=\sum_{k} u_{k}(x) \bar{u}_{k}^{\dagger}(y)$. For appropriate decreasing of $P(x, y)$ and $\bar{P}(x, y)$ for large $|y|$ also the individual terms for different $k$ decrease since they correspond to projections which are orthogonal to each other. Because this means decreasing of $u_{k}(y)$ and $\bar{u}_{k}(y)$, it becomes obvious that also $Q(x, y)$ does appropriately decrease.

The operator $Q$ related to the basis contribution in the effective action (6.21) obviously satisfies

$$
Q Q^{\dagger}=P_{-}, \quad Q^{\dagger} Q=\bar{P}_{+}, \quad P_{-} Q=Q \bar{P}_{+}=Q
$$

and describes a unitary mapping between the spaces $\mathcal{E}_{\bar{P}}$ and $\mathcal{E}_{P}$ for $\bar{N}=N$. We now observe that considering (6.22) as the defining relations of $Q$, we can avoid referring to bases at all. The question of the inequivalent subsets of bases is replaced by that of a phase factor which is left open by (6.22).

\subsection{General index and zero modes with determinant}

From the spectral representations (2.32) we have seen that $\tilde{N}=N-N_{-}(1)=\bar{N}-N_{+}(1)$, which suggests to introduce a $\tilde{N} \times \tilde{N}$ matrix $\tilde{M}$ from which in contrast to $M$ the zero modes are removed. For this purpose we introduce bases corresponding to the decomposition (2.32). Putting $P_{k}^{[-]}=\sum_{j_{k}=1}^{N_{k}} u_{j_{k}} u_{j_{k}}^{\dagger}$ we see from (2.33) that

$$
\bar{u}_{j_{k}}=\mathrm{e}^{-i \Theta_{k}}\left|f\left(\mathrm{e}^{i \varphi_{k}}\right)\right|^{-1} D u_{j_{k}}
$$


with phases $\Theta_{k}$ gives the representation $\bar{P}_{k}^{[+]}=\sum_{j_{k}=1}^{N_{k}} \bar{u}_{j_{k}} \bar{u}_{j_{k}}^{\dagger}$ and because of

$$
D^{\dagger} D=|f(-1)|^{2}\left(P_{2}^{(+)}+P_{2}^{(-)}\right)+\sum_{k\left(0<\varphi_{k}<\pi\right)}\left|f\left(\mathrm{e}^{i \varphi_{k}}\right)\right|^{2}\left(P_{k}^{(\mathrm{I})}+P_{k}^{(\mathrm{II})}\right)
$$

and $P_{k}^{(\mathrm{I})}+P_{k}^{(\mathrm{II})}=P_{k}^{[+]}+P_{k}^{[-]}=\bar{P}_{k}^{[+]}+\bar{P}_{k}^{[-]}$is properly normalized. Similarly we put $P_{2}^{(\mp)}=\sum_{j=1}^{N_{\mp}(-1)} u_{j} u_{j}^{\dagger}$, which with

$$
\bar{u}_{j}=\mathrm{e}^{-i \Theta}|f(-1)|^{-1} f(-1) u_{j}=\mathrm{e}^{-i \Theta}|f(-1)|^{-1} D u_{j}
$$

and phase $\Theta$ leads to $P_{2}^{(\mp)}=\sum_{j=1}^{N_{\mp}(-1)} \bar{u}_{j} \bar{u}_{j}^{\dagger}$. The definition of the bases then is completed introducing $u_{j_{-}}$and $\bar{u}_{j_{+}}$with $P_{1}^{(-)}=\sum_{j_{-}=1}^{N_{-}(1)} u_{j_{-}} u_{j_{-}}^{\dagger}$ and $P_{1}^{(+)}=\sum_{j_{+}=1}^{N_{+}(1)} \bar{u}_{j_{+}} \bar{u}_{j_{+}}^{\dagger}$.

The nonvanishing matrix elements of (6.3) with the above bases and identification of the indices are $M_{j_{k} j_{k}}=\mathrm{e}^{i \Theta_{k}}\left|f\left(\mathrm{e}^{i \varphi_{k}}\right)\right|$ and $M_{j j}=\mathrm{e}^{i \Theta}|f(-1)|$. We consider these elements as those of the diagonal $\tilde{N} \times \tilde{N}$ matrix $\tilde{M}$. Working out (6.5) with this we obtain for it the form ${ }^{5}$

$$
\begin{aligned}
& \left\langle\chi_{i_{r+1}} \ldots \chi_{i_{N}} \bar{\chi}_{j_{r+1}} \ldots \bar{\chi}_{j_{\bar{N}}}\right\rangle_{\mathrm{f}}=\frac{1}{(\tilde{N}-r) !} \sum_{l_{r+1}^{\prime}, \ldots, l_{\tilde{N}}^{\prime}} \sum_{l_{r+1}, \ldots, l_{\tilde{N}}} \tilde{M}_{l_{r+1}^{\prime} l_{r+1}}^{-1} \ldots \\
& \ldots \tilde{M}_{l_{\tilde{N}}^{\prime} l_{\tilde{N}}}^{-1} \epsilon_{l_{r+1} \ldots l_{\tilde{N}}, N+1, \ldots, \bar{N}}^{j_{r+1}, \ldots, j_{\bar{N}}} \epsilon_{l_{r+1} \ldots l_{\tilde{N}}, \tilde{N}+1, \ldots, N}^{k_{r+1}, \ldots, k_{N}} \operatorname{det}_{\tilde{N}} \tilde{M} \\
& \operatorname{det}_{\tilde{N}} \tilde{M}=\left(\mathrm{e}^{i \Theta}|f(-1)|\right)^{N_{\mp}(-1)} \prod_{k}\left(\mathrm{e}^{i \Theta_{k}}\left|f\left(\mathrm{e}^{i \varphi_{k}}\right)\right|\right)^{N_{k}},
\end{aligned}
$$

which with (6.1) gives

$$
\begin{aligned}
& \left\langle\psi_{\sigma_{r+1}} \ldots \psi_{\sigma_{N}} \bar{\psi}_{\left.\bar{\sigma}_{r+1} \ldots \bar{\psi}_{\bar{\sigma}_{\bar{N}}}\right\rangle_{\mathrm{f}}}\right. \\
& =\sum_{\sigma_{r+1}^{\prime}, \ldots, \sigma_{N}^{\prime}} \epsilon_{\sigma_{r+1} \ldots \sigma_{N}}^{\sigma_{r+1}^{\prime} \ldots \sigma_{N}^{\prime}} \sum_{\bar{\sigma}_{r+1}^{\prime}, \ldots, \bar{\sigma}_{\bar{N}}^{\prime}} \epsilon_{\bar{\sigma}_{r+1} \ldots \bar{\sigma}_{\bar{N}}}^{\bar{\sigma}_{r+1}^{\prime} \ldots \bar{\sigma}_{\bar{N}}^{\prime}} \frac{1}{(\tilde{N}-r) !}\left(\tilde{P}_{-} \tilde{D}^{-1} \tilde{\bar{P}}_{+}\right)_{\sigma_{r+1}^{\prime} \bar{\sigma}_{r+1}^{\prime}} \ldots \\
& \quad \ldots\left(\tilde{P}_{-} \tilde{D}^{-1} \tilde{\bar{P}}_{+}\right)_{\sigma_{\tilde{N}}^{\prime} \bar{\sigma}_{\tilde{N}}^{\prime}} u_{\sigma_{\tilde{N}+1}, \tilde{N}+1} \ldots u_{\sigma_{N} N} \bar{u}_{\tilde{N}+1, \bar{\sigma}_{\tilde{N}+1}}^{\dagger} \ldots \bar{u}_{\bar{N} \bar{\sigma}_{\bar{N}}^{\dagger}}^{\dagger} \operatorname{det}_{\tilde{N}} \tilde{M}
\end{aligned}
$$

where $\tilde{P}_{-}, \tilde{D}$ and $\tilde{\bar{P}}_{+}$are the operators $P_{-}, D$ and $\bar{P}_{+}$restricted to the subspace on which $\mathbb{1}-P_{1}^{(+)}-P_{1}^{(-)}$projects. While (6.28) resembles the conventional form (6.17), in contrast to (6.17) it allows general $N$ and $\bar{N}$ and zero modes.

Subsets of bases related to different choices of the phases $\Theta_{k}$ and $\Theta$ are obviously inequivalent if $\sum_{k} N_{k} \Theta_{k}+N_{\mp}(-1) \Theta$ gets different. The choices $\Theta_{k}=0$ and $\Theta=0$ in (6.28) corresponds to that in the generating functional of Ref. [6], which has been derived using eigenfunctions of $D D^{\dagger}$. (This functional does, however, not account for the restrictions related to the number of zero modes, which become explicit in our result (6.28) ). 


\section{Alternating-form representation}

\subsection{Structure of correlation functions}

Inserting (6.3) into (6.6) we get the form

$$
\left\langle\psi_{\sigma_{r+1}} \ldots \psi_{\sigma_{N}} \bar{\psi}_{\bar{\sigma}_{r+1}} \ldots \bar{\psi}_{\bar{\sigma}_{\bar{N}}}\right\rangle_{\mathrm{f}}=\frac{1}{r !} \sum_{\bar{\sigma}_{1} \ldots \bar{\sigma}_{r}} \sum_{\sigma_{1}, \ldots, \sigma_{r}} \bar{\Upsilon}_{\bar{\sigma}_{1} \ldots \bar{\sigma}_{\bar{N}}}^{*} \Upsilon_{\sigma_{1} \ldots \sigma_{N}} D_{\bar{\sigma}_{1} \sigma_{1}} \ldots D_{\bar{\sigma}_{r} \sigma_{r}}
$$

with the totally antisymmetric quantities

$$
\begin{aligned}
& \Upsilon_{\sigma_{1} \ldots \sigma_{N}}=\sum_{i_{1}, \ldots, i_{N}=1}^{N} \epsilon_{i_{1}, \ldots, i_{N}} u_{\sigma_{1} i_{1}} \ldots u_{\sigma_{N} i_{N}} \\
& \bar{\Upsilon}_{\bar{\sigma}_{1} \ldots \bar{\sigma}_{\bar{N}}}=\sum_{j_{1}, \ldots, j_{\bar{N}}=1}^{\bar{N}} \epsilon_{j_{1}, \ldots, j_{\bar{N}}} \bar{u}_{\bar{\sigma}_{1} j_{1}} \ldots \bar{u}_{\bar{\sigma}_{\bar{N}} j_{\bar{N}}} .
\end{aligned}
$$

For $\Upsilon_{\sigma_{1} \ldots \sigma_{N}}$ with $u$ and $\Upsilon_{\sigma_{1} \ldots \sigma_{N}}^{(S)}$ with $u^{(S)}=u S$ we obtain $\Upsilon_{\sigma_{1} \ldots \sigma_{N}}^{(S)}=\Upsilon_{\sigma_{1} \ldots \sigma_{N}} \operatorname{det}_{\mathrm{w}} S$, so that with (6.9) we have the basis independences

$$
\Upsilon_{\sigma_{1} \ldots \sigma_{N}}^{(S)}=\Upsilon_{\sigma_{1} \ldots \sigma_{N}}, \quad \bar{\Upsilon}_{\bar{\sigma}_{1} \ldots \bar{\sigma}_{\bar{N}}}^{(\bar{S})}=\bar{\Upsilon}_{\bar{\sigma}_{1} \ldots \bar{\sigma}_{\bar{N}}}
$$

For $\bar{N}=N$, in an intermediate step using $\operatorname{Tr}_{\overline{\mathrm{w}} \mathrm{w}}\left(\left(\bar{u}^{\dagger} u\right)^{\rho}\right)=\operatorname{Tr}\left((Q)^{\rho}\right)$ in the relation between determinant and traces, we obtain

$$
\frac{1}{N !} \sum_{\sigma_{1} \ldots \sigma_{N}} \bar{\Upsilon}_{\sigma_{1} \ldots \sigma_{N}}^{*} \Upsilon_{\sigma_{1} \ldots \sigma_{N}}=\operatorname{det}_{P \bar{P}}(Q)=\mathrm{e}^{\operatorname{Tr} \ln Q}
$$

by which the contribution $\operatorname{Tr} \ln Q$ of the bases in in the effective action (6.21) is expressed solely in terms of $\Upsilon_{\sigma_{1} \ldots \sigma_{N}}$ and $\bar{\Upsilon}_{\sigma_{1} \ldots \sigma_{N}}$.

It is instructive to compare the correlation function (7.1) of the chiral case with the ones of vector theory [14],

$$
\left\langle\psi_{\sigma_{r+1}} \ldots \psi_{\sigma_{K}} \bar{\psi}_{\bar{\sigma}_{r+1}} \ldots \bar{\psi}_{\bar{\sigma}_{K}}\right\rangle_{\mathrm{f}}^{\text {vect }}=\frac{1}{r !} \sum_{\bar{\sigma}_{1} \ldots \bar{\sigma}_{r}} \sum_{\sigma_{1}, \ldots, \sigma_{r}} \epsilon_{\bar{\sigma}_{1} \ldots \bar{\sigma}_{K}} \epsilon_{\sigma_{1} \ldots \sigma_{K}} D_{\bar{\sigma}_{1} \sigma_{1}} \ldots D_{\bar{\sigma}_{r} \sigma_{r}}
$$

in which $K=\operatorname{Tr} \mathbb{1}$. It is seen that, while in the vector case one has the form $\epsilon_{\sigma_{1} \ldots \sigma_{K}}$ related to full fermion space, in the chiral case one gets the forms $\Upsilon_{\sigma_{1} \ldots \sigma_{N}}$ and $\bar{\Upsilon}_{\bar{\sigma}_{1} \ldots \bar{\sigma}_{\bar{N}}}$ related to its subspaces $\mathcal{E}_{P}$ and $\mathcal{E}_{\bar{P}}$, respectively.

\subsection{Alternating multilinear forms}

An alternating multilinear form in $N$ variables is a scalar-valued function of $N$ vectors which is linear with respect to each of its arguments and vanishes if two of the arguments 
are equal. The latter implies the alternating, i.e. the change of sign if two of the arguments are interchanged. In the particular case, where $N$ is equal to the dimension of the respective vector space, such a form is completely determined [15] up to a scalar factor by its value for any set of bases taken as the arguments.

This is exactly the situation of interest here, where $\Upsilon_{\sigma_{1} \ldots \sigma_{N}}$ and $\bar{\Upsilon}_{\bar{\sigma}_{1} \ldots \bar{\sigma}_{\bar{N}}}$ in (17.2) are seen to be explicit constructions of alternating multilinear forms in the spaces $\mathcal{E}_{P}$ and $\mathcal{E}_{\bar{P}}$, respectively, the arguments of which are bases in $\sigma$-representation. The basis independence of these forms obviously realizes the general law.

This suggests to introduce $\Upsilon_{\sigma_{1} \ldots \sigma_{N}}$ and $\bar{\Upsilon}_{\bar{\sigma}_{1} \ldots \bar{\sigma}_{N}}$, which together with $D$ completely determine general correlation functions, in a slightly more general way by the relations

$$
\begin{gathered}
\Upsilon_{\sigma_{1} \ldots \sigma_{i} \ldots \sigma_{j} \ldots \sigma_{N}}=-\Upsilon_{\sigma_{1} \ldots \sigma_{j} \ldots \sigma_{i} \ldots \sigma_{N}}, \\
\sum_{\sigma_{i}}\left(P_{-}\right)_{\sigma_{j} \sigma_{i}} \Upsilon_{\sigma_{1} \ldots \sigma_{i} \ldots \sigma_{N}}=\Upsilon_{\sigma_{1} \ldots \sigma_{j} \ldots \sigma_{N}}, \\
\frac{1}{N !} \sum_{\sigma_{1}, \ldots, \sigma_{N}} \Upsilon_{\sigma_{1} \ldots \sigma_{N}}^{*} \Upsilon_{\sigma_{1} \ldots \sigma_{N}}=1,
\end{gathered}
$$

for $\Upsilon_{\sigma_{1} \ldots \sigma_{N}}$ and by analogous ones for $\bar{\Upsilon}_{\bar{\sigma}_{1} \ldots \bar{\sigma}_{\bar{N}}}$. The total antisymmetry of $\Upsilon_{\sigma_{1} \ldots \sigma_{N}}$ is imposed by (17.6). The eigenequations (7.7) determine it up to a normalization factor. The normalization then is fixed by (7.8) up to a phase factor. The choice of the latter corresponds to the selection of one of the inequivalent subsets of bases considered before.

\section{Gauge-field variations}

\subsection{Definitions and general relations}

We define general gauge variations for a function $\phi(\mathcal{U})$ by

$$
\delta \phi(\mathcal{U})=\left.\frac{\mathrm{d} \phi(\mathcal{U}(t))}{\mathrm{d} t}\right|_{t=0}, \quad \mathcal{U}_{\mu}(t)=\mathrm{e}^{t \mathcal{B}_{\mu}^{\text {left }}} \mathcal{U}_{\mu} \mathrm{e}^{-t \mathcal{B}_{\mu}^{\text {right }}}
$$

where $t$ is a real parameter and where we have

$$
\left(\mathcal{B}_{\mu}^{\text {left }}\right)_{n^{\prime} n}=B_{\mu n}^{\text {left }} \delta_{n^{\prime}, n}^{4}, \quad B_{\mu n}^{\text {left }}=i \sum_{\ell} b_{\mu n}^{\text {left } \ell} T^{\ell}
$$

and analogous relations for $\mathcal{B}_{\mu}^{\text {right }}$, with Hermitian generators $T^{\ell}$ and with $b_{n}^{\text {left } \ell}$ and $b_{n}^{\text {right } \ell}$ being real. For the variations $\delta^{\mathrm{G}}$ related to gauge transformations we get

$$
\mathcal{B}_{\mu}^{\text {left }}=\mathcal{B}_{\mu}^{\text {right }}=\mathcal{B},
$$

with $\mathcal{B}$ as already met in (4.1). 


\subsection{Variations of bases}

Varying the logarithm of $\operatorname{det}_{\mathrm{w}} S=1$ from (6.9) one gets the condition

$$
\operatorname{Tr}_{\mathrm{w}}\left(S^{\dagger} \delta S\right)=0
$$

In (8.4) the restriction to the subset of bases connected by unimodular transformations of (6.9) is lost and an extension to the larger subset with constant phase factors for the determinant occurs.

We can rewrite (8.4) in terms of bases by inserting $S=u^{\dagger} u^{(S)}$ from (15.2), which gives

$$
\operatorname{Tr}\left(\delta u^{(S)} u^{(S) \dagger}\right)=\operatorname{Tr}\left(\delta u u^{\dagger}\right)
$$

showing that the quantity $\operatorname{Tr}\left(\delta u u^{\dagger}\right)$ is basis independent within the indicated extended subset. Obviously (8.5), following from (8.4), is analogous to (7.3) for $\Upsilon_{\sigma_{1} \ldots \sigma_{N}}$ of the alternating-form representation, which follows from the original condition (6.9).

We separate the inessential dependences on $\mathcal{B}_{\mu}^{\text {left }}$ and $\mathcal{B}_{\mu}^{\text {right }}$ off, getting

$$
\operatorname{Tr}\left(\delta u u^{\dagger}\right)=2 i \operatorname{Im} \sum_{\mu, n} \operatorname{tr}_{\mathrm{g}}\left(\rho_{\mu n} \delta U_{\mu n}\right)
$$

with the variation of the field

$$
\delta U_{\mu n}=B_{\mu, n+\hat{\mu}}^{\mathrm{left}} U_{\mu n}-U_{\mu n} B_{\mu n}^{\mathrm{right}}
$$

and the quantity

$$
\rho_{\mu n, \alpha^{\prime} \alpha}=\sum_{j, \sigma} u_{j \sigma}^{\dagger} \frac{\partial u_{\sigma j}}{\partial U_{\mu n, \alpha \alpha^{\prime}}},
$$

which is invariant within the extended subset of bases. Conversely then $\rho_{\mu n}$ characterizes such a subset. It is to be noted that according to (2.29) $N$ may depend on $I$, in which case for each $I$ a different quantity $\rho_{\mu n}$ occurs.

For the basis term in the effective action (6.21), inserting $Q=u \bar{u}^{\dagger}$, we get

$$
\delta \operatorname{Tr} \ln Q=\operatorname{Tr}\left(Q^{\dagger} \delta Q\right)=\operatorname{Tr}\left(\delta u u^{\dagger}\right)-\operatorname{Tr}\left(\delta \bar{u} \bar{u}^{\dagger}\right)
$$

showing the relation to $Q$, which by (6.22) can also be defined without referring to bases. Furthermore, with (7.4) this can also be expressed in terms of $\Upsilon_{\sigma_{1} \ldots \sigma_{N}}$ and $\bar{\Upsilon}_{\sigma_{1} \ldots \sigma_{N}}$ of the alternating-form representation.

We add that also for the more general variations (8.1) the identity, used in Ref. [2],

$$
\delta_{1} \operatorname{Tr}\left(\delta_{2} u u^{\dagger}\right)-\delta_{2} \operatorname{Tr}\left(\delta_{1} u u^{\dagger}\right)+\operatorname{Tr}\left(\delta_{[2,1]} u u^{\dagger}\right)=\operatorname{Tr}\left(P_{-}\left[\delta_{1} P_{-}, \delta_{2} P_{-}\right]\right),
$$

follows readily from the defining relations (5.1) of the bases (with the generators being $\mathcal{B}_{\mu(1)}^{\text {left }}, \mathcal{B}_{\mu(1)}^{\text {right }}$ and $\mathcal{B}_{\mu(2)}^{\text {left }}, \mathcal{B}_{\mu(2)}^{\text {right }}$ and $\left[\mathcal{B}_{\mu(2)}^{\text {left }}, \mathcal{B}_{\mu(1)}^{\text {left }}\right],\left[\mathcal{B}_{\mu(2)}^{\text {right }}, \mathcal{B}_{\mu(1)}^{\text {right }}\right]$, respectively $)$. 


\subsection{Variations from gauge transformations}

For the operators $V, D, G, \bar{G}, P_{ \pm}, \bar{P}_{ \pm}$gauge transformations in (4.4) - (4.6) have been seen to have the explicit form $\mathcal{O}^{\prime}=\mathcal{T} \mathcal{O} \mathcal{T}^{\dagger}$ where $\mathcal{T}=\mathrm{e}^{\mathcal{B}}$ and $\mathcal{B}=\mathcal{B}_{\mu}^{\text {left }}=\mathcal{B}_{\mu}^{\text {right }}$. Introducing a real parameter $t$ the respective behavior can also be expressed by

$$
\mathcal{O}(\mathcal{U}(t))=\mathcal{T}(t) \mathcal{O}(\mathcal{U}(0)) \mathcal{T}^{\dagger}(t), \quad \mathcal{T}(t)=\exp (t \mathcal{B}) .
$$

The variations $\delta^{\mathrm{G}}$ corresponding to gauge transformations for such operators therefore can be obtained simply by inserting (8.11) into (8.1), with the result

$$
\delta^{\mathrm{G}} \mathcal{O}=[\mathcal{B}, \mathcal{O}] .
$$

With (8.12) we have $\delta^{\mathrm{G}} P_{-}=\left[\mathcal{B}, P_{-}\right]$for $P_{-}=u u^{\dagger}$, so that we get $\delta^{\mathrm{G}} u u^{\dagger}+u \delta^{\mathrm{G}} u^{\dagger}=$ $\mathcal{B} u u^{\dagger}-u u^{\dagger} \mathcal{B}$. This and the analogous relation for $\bar{P}_{+}$give the conditions

$$
\left(\delta^{\mathrm{G}} u-\mathcal{B} u\right) u^{\dagger}+\left(\left(\delta^{\mathrm{G}} u-\mathcal{B} u\right) u^{\dagger}\right)^{\dagger}=0, \quad\left(\delta^{\mathrm{G}} \bar{u}-\mathcal{B} \bar{u}\right) \bar{u}^{\dagger}+\left(\left(\delta^{\mathrm{G}} \bar{u}-\mathcal{B} \bar{u}\right) \bar{u}^{\dagger}\right)^{\dagger}=0,
$$

which are to be satisfied by $\delta^{\mathrm{G}} u$ and $\delta^{\mathrm{G}} \bar{u}$, however, are not sufficient to determine them.

To obtain $\delta^{\mathrm{G}} u$ and $\delta^{\mathrm{G}} \bar{u}$ we have again to resort to our knowledge from finite transformations. In the case $G \neq \mathbb{1}, \bar{G} \neq \mathbb{1}$, proceeding similarly as above for operators, (5.6) can be expressed in the form

$$
u(\mathcal{U}(t))=\mathcal{T}(t) u(\mathcal{U}(0)), \quad \bar{u}(\mathcal{U}(t))=\mathcal{T}(t) \bar{u}(\mathcal{U}(0)),
$$

which inserted into (8.1) gives

$$
\delta^{\mathrm{G}} u=\mathcal{B} u, \quad \delta^{\mathrm{G}} \bar{u}=\mathcal{B} \bar{u} \quad \text { for } \quad G \neq \mathbb{1}, \quad \bar{G} \neq \mathbb{1} .
$$

In the exceptional case $G \neq \mathbb{1}, \bar{G}=\mathbb{1}$ with (5.7) we get

$$
\delta^{\mathrm{G}} u=\mathcal{B} u, \quad \delta^{\mathrm{G}} \bar{u}_{c}=0 \quad \text { for } \quad G \neq \mathbb{1}, \quad \bar{G}=\mathbb{1} .
$$

It can be seen that (8.13) is satisfied by (8.15) and (8.16) as it must be.

It should be emphasized here that the results for the variations related to gauge transformations obviously rely entirely on the results for the finite transformations, for the operators as well as for the bases.

\subsection{Variations of effective action}

We obtain the variation of the effective action by varying our result (6.21), which gives

$$
\delta \ln \operatorname{det}_{\overline{\mathrm{w}} \mathrm{w}} M=\operatorname{Tr}\left(P_{-} D^{-1} \bar{P}_{+} \delta D+\delta u u^{\dagger}-\delta \bar{u} \bar{u}^{\dagger}\right) .
$$

For the last two terms in this we obtain with (8.6) and the analogous relation for $\bar{u}$

$$
\operatorname{Tr}\left(\delta u u^{\dagger}-\delta \bar{u} \bar{u}^{\dagger}\right)=2 i \operatorname{Im} \sum_{\mu, n} \operatorname{tr}_{\mathrm{g}}\left(\left(\rho_{\mu n}-\bar{\rho}_{\mu n}\right) \delta U_{\mu n}\right),
$$


indicating that $\rho_{\mu n}-\bar{\rho}_{\mu n}$ provides an additional term in the classical equation of motion.

Specializing (8.17) to variations related to gauge transformations, with (8.12) inserted for $\delta^{\mathrm{G}} D$, one gets

$$
\delta^{\mathrm{G}} \ln \operatorname{det}_{\overline{\mathrm{w}} \mathrm{w}} M=\operatorname{Tr}\left(\left(\bar{P}_{+}-P_{-}\right) \mathcal{B}+\delta^{\mathrm{G}} u u^{\dagger}-\delta^{\mathrm{G}} \bar{u} \bar{u}^{\dagger}\right) .
$$

For $G \neq \mathbb{1}, \bar{G} \neq \mathbb{1}$, using (8.15) and (5.1), we obtain

$$
\operatorname{Tr}\left(\delta^{\mathrm{G}} u u^{\dagger}\right)=\operatorname{Tr}\left(P_{-} \mathcal{B}\right), \quad \operatorname{Tr}\left(\delta^{\mathrm{G}} \bar{u} \bar{u}^{\dagger}\right)=\operatorname{Tr}\left(\bar{P}_{+} \mathcal{B}\right),
$$

so that in this case (8.19) vanishes, as is to be expected from the result $\ln \operatorname{det}_{\overline{\mathrm{w}} \mathrm{w}} M^{\prime}=$ $\ln \operatorname{det}_{\overline{\mathrm{w} w}} M$ for finite transformations. For $G \neq \mathbb{1}, \bar{G}=\mathbb{1}$, where according to (8.16) $\operatorname{Tr}\left(\delta^{\mathrm{G}} u u^{\dagger}\right)=\operatorname{Tr}\left(P_{-} \mathcal{B}\right)$ and $\operatorname{Tr}\left(\delta^{\mathrm{G}} \bar{u}_{c} \bar{u}_{c}^{\dagger}\right)=0$, because of $\bar{P}_{+}=\frac{1}{2}\left(1+\gamma_{5}\right) \mathbb{1}$ we get

$$
\delta^{\mathrm{G}} \ln \operatorname{det}_{\overline{\mathrm{w}} \mathrm{w}} M=\frac{1}{2} \operatorname{Tr} \mathcal{B},
$$

which is in perfect agreement with the result $\ln \operatorname{det}_{\overline{\mathrm{w} w}} M^{\prime}=\ln \operatorname{det}_{\overline{\mathrm{w} w}} M+\frac{1}{2} \operatorname{Tr} \mathcal{B}$ for finite transformations.

The term $\operatorname{Tr}\left(\left(\bar{P}_{+}-P_{-}\right) \mathcal{B}\right)$ in $(8.19)$ is the the gauge-anomaly term. To see this in detail we insert (2.17), getting

$$
\operatorname{Tr}\left(\left(\bar{P}_{+}-P_{-}\right) \mathcal{B}\right)=\frac{1}{2} \operatorname{Tr}\left(\gamma_{5} \mathcal{B}(\bar{G}+G)\right),
$$

and specialize to $\bar{G}=\mathbb{1}, G=V$, which with (4.2) gives

$$
\operatorname{Tr}\left(\left(\bar{P}_{+}-P_{-}\right) \mathcal{B}\right)=\frac{1}{2} \operatorname{Tr}\left(\gamma_{5} \mathcal{B} V\right)=i \sum_{n, \ell} b_{n}^{\ell} \frac{1}{2} \operatorname{tr}\left(\gamma_{5} T^{\ell} V_{n n}\right) .
$$

The term $\frac{1}{2} \operatorname{tr}\left(\gamma_{5} T^{\ell} V_{n n}\right)$ differs from the one of the chiral anomaly only by the insertion of the factor $T^{\ell}$. Since the inclusion of this factor in the derivation of the continuum limit is straightforward and because for the overlap $V$ this limit is safely known (see Ref. [17] for a proof and a discussion of literature) one gets

$$
\frac{1}{2} \operatorname{tr}\left(\gamma_{5} T^{\ell} V_{n n}\right) \frac{1}{a^{4}} \rightarrow-\frac{1}{32 \pi^{2}} \sum_{\mu \nu \lambda \tau} \epsilon_{\mu \nu \lambda \tau} \operatorname{tr}_{\mathrm{g}}\left(T^{\ell} F_{\mu \nu}(x) F_{\lambda \tau}(x)\right) .
$$

This still holds for the subclass of operators $D$ with $H(X)=X$ and any $W$ in (3.18) because only $V$ enters relation (2.16). It is also expected for $H(X)=X^{2 k+1}$, where the case of the chiral anomaly has been checked in Ref. [18]. For more general $V$ and for other choices of $G$ and $\bar{G}$ this limit remains to be investigated.

The r.h.s of (8.24) vanishes if the anomaly cancelation condition $\operatorname{tr}_{\mathrm{g}}\left(T^{a}\left\{T^{b}, T^{c}\right\}\right)=0$ holds, which is crucial in continuum perturbation theory. In contrast to the latter, here for $\bar{G} \neq \mathbb{1}, \bar{G} \neq \mathbb{1}$ with (8.20) the bases provide a term compensating the anomaly term at the finite stage. In the exceptional cases $\bar{G} \neq \mathbb{1}, \bar{G}=\mathbb{1}$ and $\bar{G}=\mathbb{1}, \bar{G} \neq \mathbb{1}$ the compensation is up to the constant $\frac{1}{2} \operatorname{Tr} \mathcal{B}$ and $-\frac{1}{2} \operatorname{Tr} \mathcal{B}$, respectively. This constant vanishes for $\operatorname{tr}_{\mathrm{g}} T^{\ell}=0$ (which is satisfied in the Standard Model). 


\section{Discussions of literature}

\subsection{Formulation of Lüscher}

In Ref. 2] the behavior of the effective action is investigated in the special case of GW fermions. The chiral projections there in our presentation correspond to the particular choice $G=V, \bar{G}=\mathbb{1}$ and the bases $\bar{u}$ are restricted to ones independent of the gauge field. The form of the gauge-field variations there in our notation reads $\delta U_{\mu n}=\eta_{\mu n} U_{\mu n}$. From (8.7) it is seen that with our general definition (8.1) one gets

$$
\eta_{\mu n}=B_{\mu, n+\hat{\mu}}^{\mathrm{left}}-U_{\mu n} B_{\mu n}^{\mathrm{right}} U_{\mu n}^{\dagger} .
$$

Referring to linearity a current is defined there by putting

$$
\operatorname{Tr}\left(\delta u u^{\dagger}\right)=-i \sum_{\mu, n} \operatorname{tr}_{\mathrm{g}}\left(\eta_{\mu n} j_{\mu n}\right) .
$$

In our presentation this current is explicitly given by

$$
j_{\mu n}=i\left(U_{\mu n} \rho_{\mu n}+\rho_{\mu n}^{\dagger} U_{\mu n}^{\dagger}\right),
$$

where $\rho_{\mu n}$ is the quantity (8.8). To specialize (9.2) to the case of gauge transformations in our formulation means simply to put $B_{\mu n}^{\text {left }}=B_{\mu n}^{\text {right }}=B_{n}$, which gives

$$
\operatorname{Tr}\left(\delta^{\mathrm{G}} u u^{\dagger}\right)=i \sum_{n} \operatorname{tr}_{\mathrm{g}}\left(B_{n} \sum_{\mu}\left(U_{\mu n}^{\dagger} j_{\mu n} U_{\mu n}-j_{\mu, n-\hat{\mu}}\right)\right) .
$$

With this and (4.2) one gets for (8.19) in the present special case the form

$$
\begin{gathered}
\delta^{\mathrm{G}} \ln _{\operatorname{det}_{\overline{\mathrm{w}} \mathrm{W}}} M=\frac{1}{2} \operatorname{Tr}\left(\gamma_{5} \mathcal{B} V\right)+\operatorname{Tr}\left(\delta^{\mathrm{G}} u u^{\dagger}\right)=i \sum_{n, \ell} b_{n}^{\ell} X_{n \ell} \\
X_{n \ell}=\frac{1}{2} \operatorname{tr}\left(\gamma_{5} T^{\ell} V_{n n}\right)+i \operatorname{tr}_{\mathrm{g}}\left(T^{\ell} \sum_{\mu}\left(U_{\mu n}^{\dagger} j_{\mu n} U_{\mu n}-j_{\mu, n-\hat{\mu}}\right)\right) .
\end{gathered}
$$

where tr denotess the trace in gauge-field and Dirac space only.

The strategy in Ref. 2] was to impose appropriate conditions on the current defined by (9.2), and then on the one hand side to look that it determines a set of bases and on the other that such a current exists. The relation to the subset of bases has been established requiring (8.10) for the current after introducing it by (9.2) into the terms there. ${ }^{6}$ The existence so far could not be shown for the general nonAbelian case.

The key quantity $\operatorname{Tr}\left(\delta u u^{\dagger}\right)$ of Ref. [2] is seen in (8.9) to be the variation of $\operatorname{Tr} \ln Q$, where $Q$ can be defined by (6.22) without referring to bases or may be expressed by (17.4) in terms of the quantities of the alternating-form representation. Thus instead of working

\footnotetext{
${ }^{6}$ This is a particular way to relate it to $P_{-}$. For the quantity $Q$ here (6.22) is such a relation and for $\Upsilon_{\sigma_{1} \ldots \sigma_{N}}$ we have (7.7) and for $\bar{\Upsilon}_{\bar{\sigma}_{1} \ldots \bar{\sigma}_{\bar{N}}}$ an analogon thereof for this.
} 
with variations (which causes an extension of the subset of bases and needs additional smoothness properties) it is preferable to work with $Q$, which as a unitary mapping between well defined spaces has also no existence problem.

Correspondingly also to work with the effective action itself is preferable, which we did in Subsection 6.6. It is to be remembered, however, that considering the effective action only, means to restrict to $I=0$ and absence of zero modes of $D$. Furthermore $\operatorname{Tr} \ln Q$, and thus also its variation $\operatorname{Tr}\left(\delta u u^{\dagger}\right)$, do not cover the general case. This is seen from (7.4), in which $\Upsilon_{\sigma_{1} \ldots \sigma_{N}}$ and $\bar{\Upsilon}_{\bar{\sigma}_{1} \ldots \bar{\sigma}_{\bar{N}}}$ occur only for $\bar{N}=N$, while also $\bar{N} \neq N$ is needed in general correlation functions (7.1).

While the developments in Ref. 2] clearly represent a big step forward, the fact that properties of the chiral projections cause related properties of the bases, has not been sufficiently observed there. Within this respect the exclusive use of variations there has been a disadvantage. This holds, in particular, for gauge-transformation properties, the appropriate analysis of which here has been seen to need the use of finite transformations.

The discussion of gauge invariance in Ref. 2] has been based on (9.6), presenting arguments that its contribution should vanish in the limit if the anomaly cancelation condition holds. Our result $\delta^{\mathrm{G}} \ln \operatorname{det}_{\overline{\mathrm{w}} \mathrm{W}} M=\frac{1}{2} \operatorname{Tr} \mathcal{B}$ for the special case addressed there means that $X_{n \ell}=\frac{1}{2} \operatorname{tr} T^{\ell}$ holds without a further condition at the finite stage.

The problem that one has to select one of the subsets of bases out of the inequivalent ones, which is describing physics, has been explained in Subsection 6.2. It has been pointed out to be still present in different form in the alternating-form representation with $\Upsilon_{\sigma_{1} \ldots \sigma_{N}}$ and $\bar{\Upsilon}_{\bar{\sigma}_{1} \ldots \bar{\sigma}_{\bar{N}}}$ and in the formulation with $Q$ not referring to bases. It remains, of course, there if instead of $\operatorname{Tr} \ln Q$ its variation $\operatorname{Tr}\left(\delta u u^{\dagger}\right)$ is used. In Ref. 2] this problem has not been addressed. In Ref. [19] arguments have been given that in perturbation theory the non-uniqueness of $\operatorname{Tr}\left(\delta u u^{\dagger}\right)$ would be irrelevant, while with respect to the general non-perturbative case nothing could be concluded.

\subsection{CP investigations of Fujikawa, Ishibashi and Suzuki}

In [7] Hasenfratz has observed that the divergence of (3.2) for $s=\frac{1}{2}$ is an obstacle for getting the usual behavior under CP transformations. Fujikawa et al. [6 have found that with their chiral projections, which in our notation are given by functions $G$ and $\bar{G}$ of the special form (3.2) with the more general operators $V$ from (3.4), one encounters a singularity if one tries to enforce a symmetric situation like in the continuum.

Here we have seen that actually $G$ and $\bar{G}$ must be different because of very general reasons. It has turned out that to allow for a nonvanishing index of $D$ we have to impose the basic condition (2.13). This together with the defining condition (2.22) for $D$ has lead to the requirement (2.26), which generally forbids equality of the functions $G$ and $\bar{G}$.

In the interesting investigations of $\mathrm{CP}$ properties of correlation functions in Ref. [6] the deviation from the continuum behavior found, in the notation of (3.2) is a replacement of $s$ by $1-s$. Obviously this is just the interchange of $G$ and $\bar{G}$ which we find in the general 
case here. We have seen that the interchanged choice belongs to the same $D$ and that it is a legitimate one, too.

The investigations of correlation functions in Ref. [6] are based on a generating functional which has been constructed using eigenfunctions of $D^{\dagger} D$. The content of it is similar to that of (6.28) with the special the choices $\Theta_{k}=\Theta=0$ (it does, however, not account for the restrictions related to the number of zero modes, which are explicit in our result (6.28) ). From our formulation it has become obvious that the change in the factor $|f(-1)|^{N_{\mp}(-1)}$ under the CP transformation is related to the two possible choices in (2.29), as we have discussed together with further features at the end of Subsection 4.2.

The generating functional constructed using eigenfunctions of $D^{\dagger} D$, in Ref. [6] has been subject to a non-unimodular basis transformation, considering the transformed form as the appropriate object. The motivation for this seems to be that the authors noticed the gauge invariance of their construction and that they wanted to accomodate the developments of Ref. 2]. However, actually this is a transformation from one subset of bases, in which (6.9) had to be respected, to another subset of bases, in which (6.9) must be respected, too. With each of such subsets one cannot escape gauge covariance of the correlation functions ${ }^{7}$ as we have seen here. ${ }^{8}$

\section{Comparison with continuum perturbation theory}

\subsection{General observations}

That in the lattice formulation gauge invariance is obtained at the finite stage without the anomaly cancelation condition, which is crucial for renormalizability of continuum perturbation theory, is a remarkable observation. That in the exceptional cases a constant factor is produced unless the condition $\operatorname{tr}_{\mathrm{g}} T^{\ell}=0$ holds, while this condition is not needed in continuum perturbation theory, ${ }^{9}$ is a further difference.

To assess these observations properly one has to note that at this point one actually is comparing rather different things. The formulation on the finite lattice is well defined in a unitary space. In continuum perturbation theory everything relies on the perturbation expansion with its definition in the continuum. Since continuum perturbation theory is to be taken as it is, the lattice approach has to be adapted for an adequate comparison. This means that one has to derive the related continuum perturbation theory from the general lattice formulation of chiral gauge theory and to compare the result with usual continuum perturbation theory.

In usual continuum perturbation theory the anomaly cancelation condition enters because appropriate Ward-Takahashi identities are needed in order that renormalizability can be established. These identities are related in a certain way to gauge invariance. Since

\footnotetext{
${ }^{7}$ Apart from the modification in the exceptional cases.

${ }^{8}$ We add that it is $D^{-1} \delta D$ which occurs in the variations of the reformulated basis term and of the Weyl term of the effective action as well, so that the non-locality argument in Ref. [6] does not apply.

${ }^{9}$ Though it is indeed satisfied in the Standard Model.
} 
it is quite useful to see how differences emerge also within this respect we compare the respective relations on the lattice and in the continuum before turning to the perturbation expansion. In this context we also clarify the rôle of bases in the continuum case.

\subsection{Ward-Takahashi identities}

It suffices to restrict the considerations here to fermionic correlation functions. The WardTakahashi identities of interest on the lattice for vector theory are of form

$$
\int[\mathrm{d} \bar{\psi} \mathrm{d} \psi] \mathrm{e}^{-S_{\mathrm{f}}}\left(\mathcal{O} \delta_{\mathrm{f}}^{\mathrm{G}} S_{\mathrm{f}}-\delta_{\mathrm{f}}^{\mathrm{G}} \mathcal{O}\right)=0
$$

which follows from $\int[\mathrm{d} \bar{\psi} \mathrm{d} \psi] \mathrm{e}^{-S_{\mathrm{f}}} \mathcal{O}$ by varying only the fermion fields by a gauge transformation (for which one has $\delta_{\mathrm{f}}^{\mathrm{G}} \psi=\mathcal{B} \psi, \delta_{\mathrm{f}}^{\mathrm{G}} \bar{\psi}=-\bar{\psi} \mathcal{B}$ ). This is actually just one example out of a variety of identities which on the lattice result from transformations leaving the integration measure invariant [20, 21, 22]. It should be noted that the quantity $\delta_{\mathrm{f}}^{\mathrm{G}} S_{\mathrm{f}}$ in (10.1) corresponds to a current derivative. ${ }^{10}$

Since the validity of (10.1) for $\mathcal{O}=\mathbb{1}$ is a prerequisite for its validity in the general case, in the following we consider this special case. We note that for $\mathcal{O}=\mathbb{1}$ the underlying transformation can alternatively also be interpreted as a variation of the gauge field because one has

$$
\int[\mathrm{d} \bar{\psi} \mathrm{d} \psi] \mathrm{e}^{-S_{\mathrm{f}}} \delta_{\mathrm{f}}^{\mathrm{G}} S_{\mathrm{f}}=-\delta^{\mathrm{G}} \operatorname{det} D
$$

for $S_{\mathrm{f}}=\bar{\psi} D \psi$. Thus the relations

$$
\begin{gathered}
\int[\mathrm{d} \bar{\psi} \mathrm{d} \psi] \mathrm{e}^{-S_{\mathrm{f}}} \delta_{\mathrm{f}}^{\mathrm{G}} S_{\mathrm{f}}=0, \\
\delta^{\mathrm{G}} \operatorname{det} D=0,
\end{gathered}
$$

describing current conservation in the quantized theory and gauge invariance of the determinant, respectively, represent equivalent views.

In the continuum analogon of (10.3), with $S_{\mathrm{f}}=\int \mathrm{d} x^{4} \bar{\psi} \not D \psi$ and the adjoint representation form $\mathcal{D}_{\mu}$ of the covariant derivative,

$$
\int[\mathrm{d} \bar{\psi} \mathrm{d} \psi] \mathrm{e}^{-S_{\mathrm{f}}} \sum_{\mu} \mathcal{D}_{\mu} J_{\mu}=0
$$

the formal functional integral is defined by its perturbation expansion. Correspondingly this expansion is to be checked. In this way one confirms the vanishing of (10.5), i.e. the conservation of the current $J_{\mu}^{\ell}(x)=\bar{\psi}(x) \gamma_{\mu} T^{\ell} \psi(x)$ in the quantized case.

\footnotetext{
${ }^{10}$ For example, for the Wilson action, putting $b_{n^{\prime}}^{\ell^{\prime}}=\delta_{n^{\prime} n}^{4} \delta_{\ell^{\prime} \ell}$ in $\mathcal{B}$ we obtain $\delta_{\mathrm{f}}^{\mathrm{G}} S_{\mathrm{f}}=\sum_{\mu}\left(J_{\mu n}^{\mathrm{I} \ell}-J_{\mu, n-\hat{\mu}}^{\mathrm{II} \ell}\right)$ with $J_{\mu n}^{\mathrm{I} \ell}=\frac{i}{2}\left(\bar{\psi}_{n}\left(\gamma_{\mu}-r\right) U_{\mu n}^{\dagger} T^{\ell} \psi_{n+\hat{\mu}}+\bar{\psi}_{n+\hat{\mu}}\left(\gamma_{\mu}+r\right) T^{\ell} U_{\mu n} \psi_{n}\right)$ and $J_{\mu n}^{\mathrm{II} \ell}=\frac{i}{2}\left(\bar{\psi}_{n}\left(\gamma_{\mu}-r\right) T^{\ell} U_{\mu n}^{\dagger} \psi_{n+\hat{\mu}}+\right.$ $\left.\bar{\psi}_{n+\hat{\mu}}\left(\gamma_{\mu}+r\right) U_{\mu n} T^{\ell} \psi_{n}\right)$, which corresponds to $\sum_{\mu} \mathcal{D}_{\mu} J_{\mu}(x)$ of continuum theory with the adjoint representation form $\mathcal{D}_{\mu}$ of the covariant derivative. Furthermore, e.g. for the choice $\mathcal{O}=\psi_{n^{\prime}} \bar{\psi}_{n^{\prime \prime}}$ in (10.1) one gets the lattice analogon of the familiar relation between vertex function and propagator.
} 
Switching to the chiral theory of the continuum with $S_{\mathrm{f}}=\int \mathrm{d} x^{4} \bar{\psi} \frac{1}{2}\left(1+\gamma_{5}\right) \not D \psi$ the current in (10.5) becomes $J_{\mu}^{\ell}(x)=\bar{\psi}(x) \frac{1}{2}\left(1+\gamma_{5}\right) \gamma_{\mu} T^{\ell} \psi(x)$. Then checking of the expansion, by which the formal functional integral is defined, reveals the gauge anomaly in the triangle diagrams, so that current conservation in the quantized theory requires to impose the anomaly cancelation condition.

We stress here that in this context actually also bases have to be introduced because the functional integration must be only over the occurring degrees of freedom. The reasons that the usual ignorance of this does not spoil the results are firstly that in all integration variables trading for basis transformations is possible and secondly that in the fermion loops of the expansions the bases drop out. It appears of some importance to consider these issues here in more detail.

The fermion fields are again of form $\bar{\psi}=\bar{\chi} \bar{u}^{\dagger}, \psi=u \chi$, in which the integration variables $\bar{\chi}, \chi$ are related to the degrees of freedom. The bases to be used here satisfy

$$
u_{\mathrm{co}} u_{\mathrm{co}}^{\dagger}=\frac{1}{2}\left(1-\gamma_{5}\right) \mathbb{1}, \quad \bar{u}_{\mathrm{co}} \bar{u}_{\mathrm{co}}^{\dagger}=\frac{1}{2}\left(1+\gamma_{5}\right) \mathbb{1} .
$$

With respect to the variations of the fermion fields we note that for the gauge transformations $\psi^{\prime}=\mathcal{T} \psi$ and $\bar{\psi}^{\prime}=\bar{\psi} \mathcal{T}^{\dagger}$ now trading for basis transformations can be done for both $\psi$ and $\bar{\psi}$ (which in the exceptional cases on the lattice was only possible either for $\psi$ or for $\bar{\psi}$ ). With (5.4) we get $\bar{\psi}^{\prime}=\bar{\chi} \bar{u}_{\mathrm{co}}^{\dagger} \mathcal{T}^{\dagger}=\bar{\chi} \bar{S}_{\mathcal{T}}^{\dagger} \bar{u}_{\mathrm{co}}^{\dagger}$ and similarly $\psi^{\prime}=\mathcal{T} u_{\mathrm{co}} \chi=u_{\mathrm{co}} S_{\mathcal{T}} \chi$. Introducing new integration variables $\bar{\chi}_{1}^{\prime}=\bar{\chi} \bar{S}_{\mathcal{T}}^{\dagger}$ and $\chi^{\prime}=S_{\mathcal{T}} \chi$ produces phase factors as calculated in (6.13), which here are $\mathrm{e}^{-\frac{1}{2} \operatorname{Tr} \mathcal{B}}$ and $\mathrm{e}^{\frac{1}{2} \operatorname{Tr} \mathcal{B}}$, respectively. Since these factors compensate each other the integration measure remains invariant.

In the fermion loops of the expansion the propagators are of form $u_{\mathrm{co}}^{\dagger} \not D_{0}^{-1} \bar{u}_{\mathrm{co}}$ and the vertices of form $\bar{u}_{\mathrm{co}}^{\dagger} \frac{1}{2}\left(1+\gamma_{5}\right)\left(\not D-\not D_{0}\right) u_{\mathrm{co}}$, so that only the combinations $u_{\mathrm{co}} u_{\mathrm{co}}^{\dagger}$ and $\bar{u}_{\mathrm{co}} \bar{u}_{\mathrm{co}}^{\dagger}$ occur, which according to (10.6) are the chiral projections and can be absorbed in the vertices. Thus the bases indeed drop out.

Turning now to the general formulation of the chiral theory on the lattice it is seen that the situation gets drastically different. The gauge field dependence of the bases no longer allows the trading as in the continuum (or in the exceptional cases not for both $\psi$ and $\bar{\psi}$ ). Thus nothing analogous to (10.3) can be obtained and one remains with the condition corresponding to (10.4). Furthermore, the bases no longer drop out from the loops of the perturbation expansion.

\subsection{Perturbation theory}

For the derivation of perturbation theory from the nonperturbative lattice formulation we consider the form (6.17) of the correlation functions, discussing first the factors $P_{-} D^{-1} \bar{P}_{+}$ and then turning to the chiral determinant $\operatorname{det}_{\overline{\mathrm{w}} \mathrm{w}} M$. It suffices again to restrict the considerations to the fermionic functions.

For the discussion of the factors $P_{-} D^{-1} \bar{P}_{+}$, introducing $U_{\mu n}=\exp \left(i a g \sum_{\ell} A_{\mu n}^{\ell} T^{\ell}\right)$ and the abbreviation $\mathcal{A}_{s}=a g A_{\mu n}^{\ell}$ with $s$ standing for the combination $(\mu, n, \ell)$, one can use 
the Taylor expansion

$$
f(\mathcal{A})=f(0)+\sum_{k=1}^{\infty} \frac{1}{k !} \sum_{s_{1}, \ldots, s_{k}} \mathcal{A}_{s_{1}} \ldots \mathcal{A}_{s_{k}}\left(\left.\frac{\partial^{k} f(\mathcal{A})}{\partial \mathcal{A}_{s_{1}} \ldots \partial \mathcal{A}_{s_{k}}}\right|_{\mathcal{A}=0}\right)
$$

to describe the gauge-field dependence of $V$. In the special case of the overlap Dirac operator $\mathbb{1}-V$ and of the dependence (3.2) of $G$ and $\bar{G}$ on $V$ the explicit functions which occur are those obtained and used up to second order in Refs. [12, 23. Because of $P_{-} D^{-1} \bar{P}_{+}=P_{-} D^{-1}=D^{-1} \bar{P}_{+}$, in the exceptional cases one can use the combination with the constant projection so that nothing remains to be done. Otherwise one has to consider, for example, $P_{-} D^{-1}=\frac{1}{2}\left(\mathbb{1}-\gamma_{5} G\right) D^{-1}$ in more detail, which means to inspect the limit of $G D^{-1}$. Since with the free Dirac operator $D_{0}=D-D_{\text {I }}$ we have $D^{-1}=$ $D_{0}^{-1}\left(\mathbb{1}-D_{\mathrm{I}} D^{-1}\right)$, this reduces to the consideration of $G D_{0}^{-1}$. We next remember that for the Fourier transform of $V$ in the free case one gets $\tilde{V} \rightarrow 1$ at zero and $\tilde{V} \rightarrow-1$ at the corners of the Brillouin zone [4. We now note that actually still only such contributions survive in the limit if the gauge field is present. In the combination $V D_{0}^{-1}$ then the corner contributions become suppressed so that one remains with $V D_{0}^{-1} \rightarrow D_{0}^{-1}$. With this it is obvious that for the form (3.2) of $G$ one obtains $G D_{0}^{-1} \rightarrow D_{0}^{-1}$ and thus altogether the correct limit for $P_{-} D^{-1} \bar{P}_{+}$.

It is immediately obvious that these arguments extend to further subclasses of operators constructed in Ref. 44 and also to further forms of $G(V)$ and $\bar{G}(V)$ with appropriate dependences on $V$. A general analysis for the whole class of operators within this respect remains to be performed.

To obtain the appropriate expansion ${ }^{11}$ of $\operatorname{det}_{\overline{\mathrm{w}} \mathrm{w}} M$, we start from the decomposition

$$
M=M_{0}+M_{\mathrm{I}}, \quad M_{0}=\left.M\right|_{\mathcal{U}=\mathbb{1}},
$$

and with $D_{0}=\left.D\right|_{\mathcal{U}=\mathbb{1}}, \bar{u}_{0}=\left.\bar{u}\right|_{\mathcal{U}=\mathbb{1}}$ and $u_{0}=\left.u\right|_{\mathcal{U}=\mathbb{1}}$ also have

$$
M_{0}=\bar{u}_{0}^{\dagger} D_{0} u_{0}, \quad M_{0}^{-1}=u_{0}^{\dagger} D_{0}^{-1} \bar{u}_{0}, \quad \bar{P}_{+0}=\bar{u}_{0} \bar{u}_{0}^{\dagger}, \quad P_{-0}=u_{0} u_{0}^{\dagger} .
$$

For the chiral determinant we then get

$$
\begin{gathered}
\operatorname{det}_{\overline{\mathrm{w} w}} M=\int \prod_{l=1}^{N}\left(\mathrm{~d} \bar{\chi}_{l} \mathrm{~d} \chi_{l}\right) \mathrm{e}^{-\bar{\chi} M \chi}=\left(1+\sum_{\ell=1}^{\infty} z_{\ell}\right) \operatorname{det}_{\overline{\mathrm{w} w}} M_{0}, \\
z_{\ell}=\frac{1}{\ell !} \sum_{j_{1}, \ldots, j_{\ell}=1}^{N} \sum_{k_{1}, \ldots, k_{\ell}=1}^{N} \epsilon_{j_{1}, \ldots, j_{\ell}}^{k_{1}, \ldots, k_{\ell}}\left(M_{0}^{-1} M_{\mathrm{I}}\right)_{j_{1} k_{1}} \ldots\left(M_{0}^{-1} M_{\mathrm{I}}\right)_{j_{\ell} k_{\ell}} .
\end{gathered}
$$

The latter quantitiy can be written in the form [14]

$$
z_{\ell}=\sum_{r=1}^{\ell} \frac{(-1)^{\ell+r}}{r !} \sum_{\rho_{1}=1}^{\ell-r+1} \cdots \sum_{\rho_{r}=1}^{\ell-r+1} \delta_{\ell, \rho_{1}+\ldots+\rho_{r}} \frac{t_{\rho_{1}}}{\rho_{1}} \ldots \frac{t_{\rho_{r}}}{\rho_{r}}
$$

\footnotetext{
${ }^{11}$ For our purpose it is not sufficient to make an ansatz for the effective action of form $\sum_{k=2}^{\infty} \frac{1}{k !} \sum_{s_{1}, \ldots, s_{k}} \mathcal{A}_{s_{1}} \ldots \mathcal{A}_{s_{k}} \mathcal{V}_{s_{1}, \ldots, s_{k}}$ as in Ref. [19], but explicit details are to be worked out.
} 
with fermion loops given by

$$
t_{\rho}=\operatorname{Tr}_{\mathrm{ww}}\left(\left(M_{0}^{-1} M_{\mathrm{I}}\right)^{\rho}\right)=\operatorname{Tr}\left(\left(D_{0}^{-1} \mathcal{M}\right)^{\rho}\right),
$$

being made up of free propagators $D_{0}^{-1}$ and vertices

$$
\mathcal{M}=\bar{u}_{0} M_{\mathrm{I}} u_{0}^{\dagger}
$$

With $D_{\mathrm{I}}=D-D_{0}, u_{\mathrm{I}}=u-u_{0}$ and $\bar{u}_{\mathrm{I}}=\bar{u}-\bar{u}_{0}$ we can write the latter in detail as

$$
\mathcal{M}=\bar{P}_{+0} D_{\mathrm{I}} P_{-0}+\bar{u}_{0} \bar{u}_{\mathrm{I}}^{\dagger} D u_{\mathrm{I}} u_{0}^{\dagger}+\bar{u}_{0} \bar{u}_{\mathrm{I}}^{\dagger} D P_{-0}+\bar{P}_{+0} D u_{\mathrm{I}} u_{0}^{\dagger}
$$

With respect to the term $\bar{P}_{+0} D_{\mathrm{I}} P_{-0}$ in $(10.15)$ we note that to $D_{0}^{-1} \bar{P}_{+0}$ and to $P_{-0} D_{0}^{-1}$ within (10.13) analogous considerations as in the above discussion of $P_{-} D^{-1} \bar{P}_{+}$apply, so that in the limit $\bar{P}_{+0}$ and $P_{-0}$ there can be replaced by $\frac{1}{2}\left(1+\gamma_{5}\right)$ and $\frac{1}{2}\left(1-\gamma_{5}\right)$, respectively. The fate of the other terms in (10.15) in the limit of (10.13) is determined by the behaviors of $u_{\mathrm{I}}$ and $\bar{u}_{\mathrm{I}}$. Since to $V$ in the limit only the contributions stemming from zero and from the corners of the Brillouin zone survive, the respective chiral projections become independent of the gauge field (as in the exceptional cases anyway one of them is). Accordingly describing them in the limit by constant bases one gets $u_{\mathrm{I}} \rightarrow 0$ and $\bar{u}_{\mathrm{I}} \rightarrow 0$. Then only the term $\bar{P}_{+0} D_{\mathrm{I}} P_{-0}$ of (10.15) contributes to the limit and one gets the correct vertex function at tree-graph order.

The latter observation does not yet imply that (10.13) gives the loops of usual continuum perturbation theory in the limit of vanishing lattice spacing $a \rightarrow 0$ because one-loop divergencies proportional to $1 / a$ can compensate factors of $a$ and thus possibly lead to deviations from this. An example of such a phenomenon has been given in Ref. [20]. Therefore there remains the task for future investigations to clarify whether and under which conditions one can arrive at usual perturbation theory as desirable.

A positive result of such investigations would mean that for the general formulation the anomaly cancelation condition is needed to preserve gauge invariance in the limit, too. With respect to the related mechanism it is to be noted that on the lattice with all terms of $\mathcal{M}$ being present one has gauge invariance of (10.13) (in the exceptional cases in addition requiring $\operatorname{Tr} T^{\ell}=0$ ) due to the compensation of the respective change of the first term on the r.h.s. in (10.15) by the changes of the terms there which involve $u_{\mathrm{I}}$ and $\bar{u}_{\mathrm{I}}$. However, in case of the vanishing of the latter in the limit this invariance gets lost for $a \rightarrow 0$. Furthermore, the exceptional cases then are no longer distinct from the nonexceptional one.

The mentioned constancy of the bases in the limit is actually only needed up to unimodular transformations. It has some impact on the selection of bases discussed in Subsection 6.2. To see this we consider a basis $u$ (analogous considerations apply to $\bar{u}$ ) of the general form $u=u_{\mathrm{c}} S$ where $u_{\mathrm{c}}$ is independent of the gauge field and $S$ a general unitary basis transformation. The constant bases can be represented by $u_{\mathrm{c}}=u_{\mathrm{cb}} S_{\mathrm{c}}$ where $u_{\mathrm{cb}}$ is one of them and the $S_{\mathrm{c}}$ are general constant basis transformations. The unitary transformations $S$ and $S_{\mathrm{c}}$ can be written as $S=\hat{S} \mathrm{e}^{i \phi / N}$ and $S_{\mathrm{c}}=\hat{S}_{\mathrm{c}} \mathrm{e}^{i \phi_{\mathrm{c}} / N}$ where $\hat{S}$ and 
$\hat{S}_{\mathrm{c}}$ are unimodular and one has $\mathrm{e}^{i \phi}=\operatorname{det} S$ and $\mathrm{e}^{i \phi_{\mathrm{c}}}=\operatorname{det} S_{\mathrm{c}}$. The unimodular $\hat{S}$ and $\hat{S}_{\mathrm{c}}$ are irrelevant in $\operatorname{det}_{\overline{\mathrm{w}} \mathrm{w}} M$ so that by putting $\hat{S}=\mathbb{1}$ and $\hat{S}_{\mathrm{cb}}=\mathbb{1}$ we can choose a convenient member of the respective subset (equivalence class). We then are left with $u=u_{\mathrm{cb}} \mathrm{e}^{i\left(\phi_{\mathrm{c}}+\phi\right) / N}$ in which different values of $\phi_{\mathrm{c}}+\phi$ characterize different (inequivalent) subsets of bases. We see now that to get the vanishing of $u_{\mathrm{I}}$ the characteristic quantity $\phi$ of the selected subset must be independent of the gauge field.

\section{Conclusions}

We have still extended the large class of Dirac operators decribing massless fermions on the lattice we have found recently, only requiring that such operators decompose into Weyl operators. Using the spectral representations of the operators we have obtained a basic condition on the Dirac operator, a general sum rule, a general expression for the index, a basic condition which prevents symmetry between the chiral projections and the detailed structure of the chiral projections. Our general construction of operators, using the tool of spectral functions, has been seen to extend, too. This also holds for the related realizations of the basic unitary operator, for which in addition some more freedom has been observed.

After making sure about the transformation properties of our general operators and performing a careful study of the basis representations of the chiral projections, we have turned to the correlation functions of Weyl fermions. For their investigation we have introduced a formulation of the fermionic functions which works for any value of the index. For the additional conditions due to the requirement of their invariance under basis transformations the consequences have been made precise. In this context we have stressed that, since formulations in different ones of the emerging subsets of bases are not equivalent, a criterion is needed telling which one of such subsets describes physics.

Considering gauge transformation the crucial importance of using finite transformations in the analysis has become obvious. We have seen that the correlation functions exhibit gauge-covariant behavior of the fermion fields. In the exceptional cases in addition constant phase factors have turned out to occur, the values of which have been determined. The behavior under CP transformations has been found to differ from that known from continuum theory by involving an interchange of the functions in the chiral projections, where the interchanged choice is a legitimate one, too. In view of our result that such functions must be generally different, we have also studied the effects of the interchange.

We have derived the explicit form of the effective action, in which the contributions of the Weyl operator and of the bases are separated. This form has allowed us to introduce a formulation not referring to bases and to discuss locality properties. Starting from the observation that for any value of the index the removal of zero modes makes the remaining chiral matrix quadratic, we have used the spectral representations to get a form of general correlation functions with a reduced chiral determinant. We have reformulated the correlation functions so that they are completely determined by alternating multilinear forms and $D$ and discussed the features of this presentation. 
Variations of the gauge fields have been defined with left and right generators. Their application to specify basis-independent quantities has been discussed and the extension of the related subsets beyond that of the unimodular case noted. The properties of variations related to gauge transformations have been obtained from those of the finite transformations and been seen to rely entirely on the latter. Considerations of the variations of the effective action have allowed various comparisons.

The developments in Ref. [2] and the investigations of CP properties of Ref. [6] have been discussed in the light of our results. A comparison with continuum perturbation theory has included the discussion of related Ward-Takahashi identities, the derivation

of perturbative results on the basis of the present nonperturbative definitions and the discussion of the relevant conditions.

\section{Acknowledgement}

I wish to thank Michael Müller-Preussker and his group for their kind hospitality.

\section{References}

[1] R. Narayanan, H. Neuberger, Phys. Rev. Lett. 71 (1993) 3251;

Nucl. Phys. B412 (1994) 574; Nucl. Phys. B443 (1995) 305.

[2] M. Lüscher, Nucl. Phys. B549 (1999) 295; Nucl. Phys. B568 (2000) 162.

[3] P.H. Ginsparg, K.G. Wilson, Phys. Rev. D25 (1982) 2649.

[4] W. Kerler, Nucl. Phys. B646 (2002) 201.

[5] K. Fujikawa, Nucl. Phys. B589 (2000) 487.

[6] K. Fujikawa, M. Ishibashi, H. Suzuki Phys. Lett. B538 (2002) 197; JHEP 0204 (2002) 046.

[7] P. Hasenfratz, Nucl. Phys. (Proc. Suppl.) 106 (2002) 159.

[8] T.-W. Chiu, Phys. Rev. D58 (1998) 074511.

[9] P. Hasenfratz, V. Laliena, F. Niedermayer, Phys. Lett. B427 (1998) 125.

[10] M. Lüscher, Phys. Lett. B428 (1998) 342.

[11] H. Neuberger, Phys. Lett. B417 (1998) 141; Phys. Lett. B427 (1998) 353.

[12] Y. Kikukawa, A. Yamada, Phys. Lett. B448 (1998) 256. 
[13] T. Kato, Perturbation theory for linear operators (Springer, Berlin · Heidelberg · New York 1966).

[14] W. Kerler, Z. Physik C22 (1984) 185.

[15] J. Dieudonné, Foundations of modern analysis, Vol. 10-I in Pure and Applied Mathematics, eds. P.A. Smith, S. Eilenberg (Academic Press, New York · London, 1969).

[16] H. Suzuki, JHEP 0010 (2000) 039.

[17] W. Kerler, Int. J. Mod. Phys. A16 (2001) 3117.

[18] K. Fujikawa and M. Ishibashi, Nucl. Phys. B587 (2000) 419.

[19] M. Lüscher, JHEP 0006 (2000) 028.

[20] L.H. Karsten, J. Smit, Nucl. Phys. B144 (1978) 536.

[21] L.H. Karsten, J. Smit, Nucl. Phys. B183 (1981) 103.

[22] W. Kerler, Phys. Rev. D24 (1981) 1595; Phys. Lett. B100 (1981) 267; Phys. Rev. D23 (1981) 2384.

[23] C. Alexandrou, H. Panagopoulos, E. Vicari, Nucl. Phys. B571 (2000) 257; M. Ishibashi, Y. Kikukawa, T. Noguchi, A. Yamada, Nucl. Phys. B576 (2000) 501. 